\title{
Supporting Information for ja060832i Quantitative Structure-Activity Relationships of Ruthenium Catalysts for Olefin Metathesis
}

\author{
Giovanni Occhipinti, Hans-René Bjørsvik and Vidar R. Jensen* \\ Department of Chemistry, University of Bergen, Allégaten 41, N-5007 Bergen, Norway.
}

\begin{tabular}{|c|c|}
\hline Table of contents & Page \\
\hline Complete reference 37 & S1 \\
\hline Table S1. List of 122 molecular descriptors & S2 \\
\hline Table S2. Numerical values of 11 important molecular descriptors for 86 Ru-complexes $\left(\mathrm{LCl}_{2} \mathrm{Ru}=\mathrm{CH}_{2}\right)$ & S5 \\
\hline Table S3. Predicted and explicitly calculated productivities for 77 Ru-complexes of ligands (L) & S7 \\
\hline Figure S1. Dendrogram showing the cluster analysis (k-means nearest group) & S8 \\
\hline Additional results from PCA for $\mathbf{X}_{77 \times 122}$ ( $77 \mathrm{LCl}_{2} \mathrm{Ru}=\mathrm{CH}_{2}$ complexes and 122 molecular descriptors) & S9 \\
\hline Table S4. Percent of variance captured by PCA model & S9 \\
\hline Figure S2. Eigenvalues plot for $\mathbf{X}_{77 \times 122}$ & S9 \\
\hline Scores plots - PCA of $\mathbf{X}_{77 \times 122}$ & S10 \\
\hline $2 \mathrm{D}$ scores plot $\mathrm{t}_{1} \mathrm{vs} \mathrm{t}_{2}$ & S10 \\
\hline $2 \mathrm{D}$ scores plot $t_{1}$ vs $t_{3}$ & S11 \\
\hline $2 \mathrm{D}$ scores plot $t_{1}$ vs $t_{4}$ & S12 \\
\hline $2 \mathrm{D}$ scores plot $\mathrm{t}_{1}$ vs $t_{5}$ & S13 \\
\hline $2 \mathrm{D}$ scores plot $t_{1}$ vs $t_{6}$ & S14 \\
\hline $2 \mathrm{D}$ scores plot $\mathrm{t}_{3} \mathrm{vs}_{2}$ & S15 \\
\hline $3 D$ scores plot $t_{1}$ vs $t_{2}$ vs $t_{3}$ & S16 \\
\hline Table S5. Score values achieved from principal component analysis (PCA) of $\mathbf{X}_{77 \times 122}$ & S17 \\
\hline Loadings plots - PCA of $\mathbf{X}_{77 \times 122}$ & S19 \\
\hline $2 D$ loadings plot $p_{1}$ vs $p_{2}$ & S19 \\
\hline $2 D$ loadings plot $p_{1}$ vs $p_{3}$ & S20 \\
\hline $2 D$ loadings plot $p_{1}$ vs $p_{4}$ & S21 \\
\hline $2 D$ loadings plot $p_{1}$ vs $p_{5}$ & S22 \\
\hline $2 D$ loadings plot $p_{1}$ vs $p_{6}$ & S23 \\
\hline $2 D$ loadings plot $p_{3}$ vs $p_{2}$ & S24 \\
\hline $3 D$ loadings plot $p_{1}$ vs $p_{2}$ vs $p_{3}$ & S25 \\
\hline Table S6. Loading values achieved from principal component analysis (PCA) of $\mathbf{X}_{77}$ & S26 \\
\hline
\end{tabular}

\section{Complete reference 37.}

Frisch, M. J.; Trucks, G. W.; Schlegel, H. B.; Scuseria, G. E.; Robb, M. A.; Cheeseman, J. R.; J. A. Montgomery, J.; Vreven, T.; Kudin, K. N.; Burant, J. C.; Millam, J. M.; Iyengar, S. S.; Tomasi, J.; Barone, V.; Mennucci, B.; Cossi, M.; Scalmani, G.; Rega, N.; Petersson, G. A.; Nakatsuji, H.; Hada, M.; Ehara, M.; Toyota, K.; Fukuda, R.; Hasegawa, J.; Ishida, M.; Nakajima, T.; Honda, Y.; Kitao, O.; Nakai, H.; Klene, M.; Li, X.; Knox, J. E.; Hratchian, H. P.; Cross, J. B.; Adamo, C.; Jaramillo, J.; Gomperts, R.; Stratmann, R. E.; Yazyev, O.; Austin, A. J.; Cammi, R.; Pomelli, C.; Ochterski, J. W.; Ayala, P. Y.; Morokuma, K.; Voth, G. A.; Salvador, P.; Dannenberg, J. J.; Zakrzewski, V. G.; Dapprich, S.; Daniels, A. D.; Strain, M. C.; Farkas, O.; Malick, D. K.; Rabuck, A. D.; Raghavachari, K.; Foresman, J. B.; Ortiz, J. V.; Cui, Q.; Baboul, A. G.; Clifford, S.; Cioslowski, J.; Stefanov, B. B.; Liu, G.; Liashenko, A.; Piskorz, P.; Komaromi, I.; Martin, R. L.; Fox, D. J.; Keith, T.; AlLaham, M. A.; Peng, C. Y.; Nanayakkara, A.; Challacombe, M.; Gill, P. M. W.; Johnson, B.; Chen, W.; Wong, M. W.; Gonzalez, C.; Pople, J. A. Gaussian 03; Revision B.04; Gaussian, Inc.: Pittsburg PA, 2003. 
Table S1. Complete list of 122 molecular descriptors used in PCA and cluster analysis.

1 Number of atoms

2 Number of $\mathrm{C}$ atoms

3 Relative number of $\mathrm{C}$ atoms

4 Number of $\mathrm{H}$ atoms

5 Relative number of $\mathrm{H}$ atoms

6 Relative number of single bonds

7 Number of aromatic bonds

8 Relative molecular weight

9 Gravitation index (all bonds)

10 Gravitation index (all pairs)

11 Wiener index

12 Kier \& Hall index (order 0)

13 Kier \& Hall index (order 1)

14 Kier \& Hall index (order 2)

15 Kier \& Hall index (order 3)

16 Kier shape index (order 1)

17 Kier shape index (order 2)

18 Kier flexibility index

19 Average Information content (order 0)

20 Average Structural Information content (order 0)

21 Complementary Information content (order 0)

22 Average Information content (order 1)

23 Average Structural Information content (order 1)

24 Complementary Information content (order 1)

25 Average Information content (order 2)

26 Average Structural Information content (order 2)

27 Structural Information content (order 2)

28 Complementary Information content (order 2)

29 Moment of inertia A

30 Moment of inertia B

31 XY Shadow / XY Rectangle

32 YZ Shadow

33 YZ Shadow / YZ Rectangle

34 ZX Shadow / ZX Rectangle

35 Molecular volume / XYZ Box

36 No. of occupied electronic levels

37 No. of occupied electronic levels / of atoms

38 HOMO-1 energy

39 HOMO energy

40 LUMO energy 


\section{Continuation of Table S1}

41 LUMO+1 energy

42 HOMO-LUMO energy gap

43 Min nucleoph. react. index for a $\mathrm{C}$ atom

44 Max nucleoph. react. index for a $\mathrm{C}$ atom

45 Avg nucleoph. react. index for a $\mathrm{C}$ atom

46 Min nucleoph. react. index for a $\mathrm{Ru}$ atom

47 Min electroph. react. index for a $\mathrm{C}$ atom

48 Max electroph. react. index for a $\mathrm{C}$ atom

49 Avg electroph. react. index for a $\mathrm{C}$ atom

50 Min electroph. react. index for a $\mathrm{Ru}$ atom

51 Min 1-electron react. index for a $\mathrm{C}$ atom

52 Max 1-electron react. index for a $\mathrm{C}$ atom

53 Avg 1-electron react. index for a $\mathrm{C}$ atom

54 Min 1-electron react. index for a $\mathrm{Ru}$ atom

55 Total dipole of the molecule (NBO)

56 Tot point-charge comp. of the molecular dipole

57 Tot hybridization comp. of the molecular dipole

58 TMSA Total molecular surface area

59 PPSA-1 Partial positive surface area

60 PNSA-1 Partial negative surface area

61 DPSA-1 Difference in CPSAs (PPSA1-PNSA1)

62 FPSA-1 Fractional PPSA (PPSA-1/TMSA)

63 FNSA-1 Fractional PNSA (PNSA-1/TMSA)

64 WPSA-1 Weighted PPSA (PPSA1*TMSA/1000)

65 WNSA-1 Weighted PNSA (PNSA1*TMSA/1000)

66 PPSA-2 Total charge weighted PPSA

67 FPSA-2 Fractional PPSA (PPSA-2/TMSA)

68 FNSA-3 Fractional PNSA (PNSA-3/TMSA)

69 RPCS Relative positive charged SA (SAMPOS*RPCG)

70 RNCG Relative negative charge (QMNEG/QTMINUS)

71 RNCS Relative negative charged SA (SAMNEG*RNCG)

72 Avg valency of a $\mathrm{H}$ atom

73 Max bond order of a $\mathrm{H}$ atom

74 Min valency of a $\mathrm{C}$ atom

75 Max valency of a $\mathrm{C}$ atom

76 Avg valency of a $\mathrm{C}$ atom

77 Min (0.1) bond order of a $\mathrm{C}$ atom

78 Max bond order of a $\mathrm{C}$ atom

79 Avg bond order of a $\mathrm{C}$ atom

80 Min valency of a $\mathrm{Ru}$ atom 


\section{Continuation of Table S1}

81 Min bond order of a $\mathrm{Ru}$ atom including contributions $>0.1$

82 Max bond order of a $\mathrm{Ru}$ atom

83 Avg bond order of a Ru atom

84 Zero point vibrational energy

85 Zero point vibrational energy / of atoms

86 Lowest normal mode vib frequency

87 Highest normal mode vib frequency

88 Absolute electronegativity: $-\left(\varepsilon_{\mathrm{HOMO}}+\varepsilon_{\mathrm{LUMO}}\right) / 2$

89 Hardness: $\left(\varepsilon_{\text {LUMO }}-\varepsilon_{\text {HOMO }}\right) / 2$

$90 \mathrm{Ru}=\mathrm{CH}_{2}$ bond length

91 Absolute value of the torsion $\mathrm{LRuCH}_{\mathrm{a}}\left(\mathrm{H}_{\mathrm{a}}\right.$ is the alkylidene hydrogen atom closest to the ligand $\left.\mathrm{L}\right)$

$92 \mathrm{~L}-\mathrm{Ru}=\mathrm{C}$ angle

$93 \mathrm{Ru}=\mathrm{C}-\mathrm{H}_{\mathrm{a}}$ angle $\left(\mathrm{H}_{\mathrm{a}}\right.$ is the hydrogen atom closest to the ligand $\left.\mathrm{L}\right)$

$94 \mathrm{H}_{\mathrm{a}}-\mathrm{C}-\mathrm{H}_{\mathrm{b}}$ angle (alkylidene moiety)

95 Angle between bisector $\mathrm{H}_{\mathrm{a}}-\mathrm{C}-\mathrm{H}_{\mathrm{b}}$ and direction $\mathrm{Ru}=\mathrm{C}$

96 Wiberg bond index: average of the bonds $\mathrm{Ru}-\mathrm{Cl}_{\mathrm{a}}$ and $\mathrm{Ru}-\mathrm{Cl}_{\mathrm{b}}$

97 Wiberg bond index, total by atom (for alkylidene $\mathrm{C}$ )

98 Atom-atom overlap-weighted NAO bond order (NBO) for Ru and alkylidene C

99 Atom-atom overlap-weighted NAO bond order, total by atom (NBO) (for alkylidene C)

100 Nucleophile Fukui index for the alkylidene C

101 Electrophile Fukui index for the alkylidene $\mathrm{C}$

102 One electron Fukui index for the alkylidene $\mathrm{C}$

103 Steric exchange energy between $\mathrm{L}$ and the $=\mathrm{CH} 2$ group (1)

104 Steric exchange energy between $\mathrm{L}$ and the $\mathrm{Cl}$ atoms of the $\mathrm{Cl}_{2} \mathrm{Ru}=\mathrm{CH}_{2}$ group (2)

105 Sum of steric exchange energy (1) and (2)

$106 \sigma$-donation L-Ru: [ $\pi$-back-donation $\mathrm{Ru}-\mathrm{L}$ ] - [CHELPG charge on fragment $\mathrm{Cl}_{2} \mathrm{Ru}=\mathrm{CH}_{2}$ ]

$107 \pi$-back-donation Ru-L

$108 \sigma$ bond order $\mathrm{Ru}=\mathrm{CH}_{2}:\left(\sigma \mathrm{NBO}\right.$ pop. $-\sigma^{*} \mathrm{NBO}$ pop. $) / 2$

$109 \pi$ bond order $\mathrm{Ru}=\mathrm{CH}_{2}:\left(\pi \mathrm{NBO}\right.$ pop. $-\pi^{*} \mathrm{NBO}$ pop. $) / 2$

110 Tot bond order $\mathrm{Ru}=\mathrm{CH}_{2}: \sigma$ bond order $\mathrm{Ru}=\mathrm{CH}_{2}+\pi$ bond order $\mathrm{Ru}=\mathrm{CH}_{2}$

111 CHELPG charge on $\mathrm{Ru}$ atom

112 CHELPG charge on alkylidene $\mathrm{C}$

113 CHELPG charge on the group $\mathrm{Cl}_{2} \mathrm{Ru}=\mathrm{CH}_{2}$

114 CHELPG charge on alkylidene group $\left(\mathrm{CH}_{2}\right)$

115 CHELPG charge on the atom of $\mathrm{L}$ that is bound to the $\mathrm{Ru}$

116 CHELPG charge average on the $\mathrm{Cl}$ belonging to the $\mathrm{Cl}_{2} \mathrm{RuCH}_{2}$ group

117 Total dipole moment (CHELPG)

118 Electrostatic potential on $\mathrm{Ru}$ atom

119 Electrostatic potential on alkylidene $\mathrm{C}$ atom

120 Electrostatic potential: average on $\mathrm{Cl}_{2} \mathrm{RuCH}_{2}$ group

121 Electrostatic potential: average on $\mathrm{CH}_{2}$ group

122 Electrostatic potential: average on the $\mathrm{Cl}$ atom belonging to the $\mathrm{Cl}_{2} \mathrm{RuCH}_{2}$ group 
Table S2. Numerical values of 11 important molecular descriptors for 86 Ru-complexes $(\mathrm{LCl} 2 \mathrm{Ru}=\mathrm{CH} 2)$.

\begin{tabular}{|c|c|c|c|c|c|c|c|c|c|c|c|}
\hline \multirow[b]{2}{*}{$L^{b}$} & \multicolumn{10}{|c|}{ Selected molecular descriptors for $\mathrm{LCl}_{2} \mathrm{Ru}=\mathrm{CH}_{2}{ }^{a}$} & \multirow[b]{2}{*}{ md117 } \\
\hline & md50 & md82 & md90 & md92 & md96 & md103 & md106 & md107 & md113 & md116 & \\
\hline A1 & 0.037 & 1.636 & 1.824 & 94.4 & 0.497 & 2.9 & 0.46 & 0.15 & -0.31 & -0.463 & 7.1 \\
\hline A2 & 0.038 & 1.644 & 1.822 & 95.0 & 0.493 & 2.3 & 0.46 & 0.14 & -0.32 & -0.474 & 6.7 \\
\hline A3 & 0.030 & 1.637 & 1.820 & 97.5 & 0.508 & 8.7 & 0.44 & 0.16 & -0.29 & -0.463 & 7.4 \\
\hline A4 & 0.032 & 1.639 & 1.821 & 96.4 & 0.510 & 7.6 & 0.43 & 0.14 & -0.29 & -0.461 & 7.1 \\
\hline A5 & 0.014 & 1.639 & 1.819 & 99.2 & 0.501 & 10.0 & 0.48 & 0.15 & -0.33 & -0.456 & 7.1 \\
\hline A6 & 0.018 & 1.641 & 1.820 & 97.5 & 0.506 & 8.7 & 0.43 & 0.13 & -0.30 & -0.457 & 6.9 \\
\hline A7 & 0.035 & 1.659 & 1.814 & 111.6 & 0.471 & 20.0 & 0.47 & 0.12 & -0.35 & -0.480 & 5.9 \\
\hline A8 & 0.014 & 1.616 & 1.817 & 98.0 & 0.480 & 17.1 & 0.52 & 0.19 & -0.33 & -0.468 & 7.2 \\
\hline A9 & 0.026 & 1.639 & 1.819 & 98.8 & 0.499 & 10.6 & 0.49 & 0.15 & -0.34 & -0.454 & 6.9 \\
\hline A10 & 0.022 & 1.640 & 1.820 & 96.9 & 0.505 & 9.4 & 0.45 & 0.13 & -0.32 & -0.452 & 6.8 \\
\hline A11 & 0.015 & 1.631 & 1.819 & 99.7 & 0.500 & 8.3 & 0.54 & 0.18 & -0.36 & -0.448 & 5.3 \\
\hline A12 & 0.035 & 1.651 & 1.816 & 104.0 & 0.491 & 9.0 & 0.51 & 0.17 & -0.34 & -0.473 & 6.9 \\
\hline A13 & 0.035 & 1.656 & 1.817 & 103.2 & 0.493 & 8.1 & 0.49 & 0.16 & -0.33 & -0.467 & 6.5 \\
\hline A14 & 0.035 & 1.646 & 1.817 & 103.6 & 0.498 & 8.9 & 0.49 & 0.17 & -0.32 & -0.454 & 4.2 \\
\hline A15 & 0.037 & 1.639 & 1.817 & 103.8 & 0.497 & 9.8 & 0.47 & 0.19 & -0.28 & -0.451 & 6.7 \\
\hline A16 & 0.031 & 1.646 & 1.817 & 104.4 & 0.489 & 8.7 & 0.54 & 0.19 & -0.36 & -0.477 & 7.5 \\
\hline A17 & 0.034 & 1.659 & 1.816 & 103.9 & 0.490 & 7.7 & 0.51 & 0.15 & -0.36 & -0.478 & 7.5 \\
\hline A18 & 0.034 & 1.647 & 1.816 & 104.0 & 0.498 & 8.3 & 0.47 & 0.14 & -0.33 & -0.438 & 4.2 \\
\hline A19 & 0.035 & 1.649 & 1.816 & 103 & & 7.8 & 0.4 & 0.14 & -0.34 & -0.463 & 6.2 \\
\hline A20 & 0.035 & 1.651 & 1.816 & 105.3 & 0.482 & 9.1 & 0.50 & 0.17 & -0.33 & -0.480 & 7.3 \\
\hline A21 & 0.035 & 1.657 & 1.816 & 103.2 & 0.491 & 8.2 & 0.49 & 0.16 & -0.33 & -0.468 & 6.5 \\
\hline A22 & 0.033 & 1.657 & 1.815 & 104.9 & 0.4 & 8.8 & 0.52 & 0.15 & -0.36 & -0.479 & 7.4 \\
\hline A23 & 0.036 & 1.643 & 1.816 & 103.4 & 0.5 & $7 . \varsigma$ & 0.46 & 0.15 & -0.31 & -0.453 & 8.1 \\
\hline A24 & 0.040 & 1.643 & 1.819 & 100.3 & 0.499 & 9.5 & 0.48 & 0.15 & -0.33 & -0.474 & 8.6 \\
\hline A25 & 0.034 & 1.658 & 1.816 & 103.0 & 0.489 & 8.5 & 0.51 & 0.15 & -0.36 & -0.475 & 5.9 \\
\hline A26 & 0.055 & 1.639 & 1.822 & 95.8 & 0.505 & 9.2 & 0.43 & 0.13 & -0.30 & -0.462 & 6.8 \\
\hline A27 & 0.022 & 1.652 & 1.817 & 95 & 0.4 & 9.1 & 0.49 & 0.12 & -0.36 & -0.468 & 5.0 \\
\hline A28 & 0.009 & 1.597 & 1.823 & 97.9 & 0.531 & 6.1 & 0.49 & 0.27 & -0.22 & -0.434 & 6.4 \\
\hline A29 & 0.014 & 1.626 & 1.819 & 99.7 & 0.505 & 7.6 & 0.50 & 0.19 & -0.31 & -0.444 & 2.9 \\
\hline A30 & 0.017 & 1.630 & 1.824 & 94.9 & 0.515 & 6.4 & 0.42 & 0.14 & -0.28 & -0.462 & 8.1 \\
\hline A31 & 0.032 & 1.618 & 1.819 & 103.5 & 0.5 & 9.8 & 051 & 0.25 & -0.25 & 31 & 4.0 \\
\hline A32 & 0.063 & 1.606 & 1.823 & 95.7 & 0.521 & 8.1 & 0.39 & 0.22 & -0.16 & -0.408 & 7.6 \\
\hline A33 & 0.007 & 1.615 & 1.819 & 104.0 & 0.506 & 10.3 & 0.51 & 0.25 & -0.26 & -0.431 & 2.6 \\
\hline A34 & 0.024 & 1.595 & 1.822 & 102.7 & 0.534 & 7.5 & 0.48 & 0.27 & -0.21 & -0.393 & 3.9 \\
\hline A35 & 0.045 & 1.612 & 1.822 & 96.0 & 0.5 & 7.8 & 0.43 & 0.21 & -0.22 & -0.431 & 7.1 \\
\hline A36 & 0.000 & 1.594 & 1.828 & 94 & & 5. & 0.3 & 0.19 & -0.17 & -0.393 & 7.2 \\
\hline A37 & 0.035 & 1.617 & 1.819 & 99.2 & 0.505 & 9.3 & 0.46 & 0.21 & -0.25 & -0.442 & 7.1 \\
\hline A38 & 0.029 & 1.617 & 1.819 & 103.2 & 0.505 & 8.5 & 0.51 & 0.25 & -0.26 & -0.438 & 4.0 \\
\hline $\mathrm{A} 39^{\mathrm{C}}$ & 0.036 & 1.654 & 1.815 & 106.6 & 0.479 & 10.6 & 0.61 & 0.17 & -0.44 & -0.480 & 7.1 \\
\hline $\mathrm{A} 40^{\mathrm{C}}$ & 0.035 & 1.651 & 1.815 & 107.6 & 0.470 & 13.1 & 0.62 & 0.16 & -0.46 & -0.478 & 6.9 \\
\hline $\mathrm{A} 41^{\mathrm{C}}$ & 0.034 & 1.659 & 1.815 & 107.2 & 0.475 & 13.2 & 0.59 & 0.14 & -0.45 & -0.470 & 6.8 \\
\hline $\mathrm{A} 42^{\mathrm{C}}$ & 0.034 & 1.652 & 1.816 & 105.8 & 0.481 & 11.2 & 0.56 & 0.17 & -0.38 & -0.473 & 7.2 \\
\hline- & & & & & & 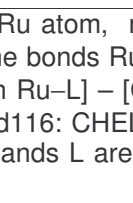 & 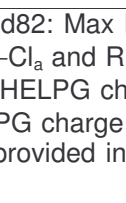 & 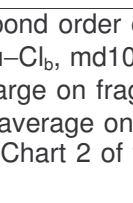 & ח & & $\begin{array}{l}\mathrm{H}_{2} \text { bond } \\
\text { between } \\
\pi \text {-back- }\end{array}$ \\
\hline
\end{tabular}


Continuation of Table S2.

\begin{tabular}{|c|c|c|c|c|c|c|c|c|c|c|c|}
\hline \multirow[b]{2}{*}{$\mathrm{L}$} & \multicolumn{10}{|c|}{ Selected molecular descriptors for $\mathrm{LCl}_{2} \mathrm{Ru}=\mathrm{CH}_{2}{ }^{\mathrm{a}}$} & \multirow[b]{2}{*}{ md117 } \\
\hline & md50 & md82 & md90 & md92 & md96 & md103 & md106 & md107 & $\mathrm{md113}$ & md116 & \\
\hline $\mathrm{P} 1$ & 0.069 & 1.604 & 1.824 & 94.2 & 0.543 & 3.3 & 0.32 & 0.08 & -0.24 & -0.438 & 5.0 \\
\hline P2 & 0.062 & 1.625 & 1.824 & 96.1 & 0.519 & 2.6 & 0.45 & 0.06 & -0.38 & -0.448 & 6.3 \\
\hline P3 & 0.060 & 1.630 & 1.822 & 97.4 & 0.512 & 3.4 & 0.42 & 0.06 & -0.36 & -0.453 & 6.5 \\
\hline P4 & 0.059 & 1.621 & 1.822 & 98.2 & 0.516 & 7.6 & 0.41 & 0.06 & -0.35 & -0.443 & 7.0 \\
\hline P5 & 0.058 & 1.633 & 1.820 & 97.3 & 0.509 & 7.6 & 0.38 & 0.06 & -0.32 & -0.460 & 6.9 \\
\hline P6 & 0.051 & 1.641 & 1.819 & 98.4 & 0.516 & 4.4 & 0.36 & 0.06 & -0.31 & -0.452 & 6.9 \\
\hline P7 & 0.042 & 1.641 & 1.819 & 100.7 & 0.501 & 6.0 & 0.38 & 0.06 & -0.32 & -0.462 & 6.8 \\
\hline P8 & 0.061 & 1.607 & 1.825 & 95.8 & 0.522 & 5.1 & 0.38 & 0.07 & -0.31 & -0.444 & 7.0 \\
\hline P9 & 0.057 & 1.612 & 1.822 & 97.2 & 0.516 & 10.5 & 0.39 & 0.06 & -0.32 & -0.459 & 6.9 \\
\hline P10 & 0.047 & 1.621 & 1.824 & 95.8 & 0.516 & 3.3 & 0.39 & 0.07 & -0.31 & -0.447 & 6.6 \\
\hline $\mathrm{P} 11$ & 0.051 & 1.617 & 1.824 & 95.4 & 0.516 & 3.6 & 0.40 & 0.07 & -0.34 & -0.445 & 6.9 \\
\hline P12 & 0.055 & 1.628 & 1.824 & 96.2 & 0.513 & 2.8 & 0.41 & 0.07 & -0.35 & -0.455 & 6.3 \\
\hline P13 & 0.066 & 1.584 & 1.828 & 93.6 & 0.537 & 2.8 & 0.33 & 0.11 & -0.23 & -0.409 & 4.5 \\
\hline P14 & 0.064 & 1.587 & 1.827 & 93.5 & 0.535 & 2.8 & 0.32 & 0.10 & -0.22 & -0.422 & 5.0 \\
\hline P15 & 0.064 & 1.576 & 1.829 & 93.7 & 0.541 & 3.2 & 0.36 & 0.12 & -0.24 & -0.419 & 3.9 \\
\hline P16 & 0.082 & 1.539 & 1.834 & 94.5 & 0.581 & 2.3 & 0.18 & 0.17 & -0.02 & -0.345 & 3.3 \\
\hline $\mathrm{N} 1$ & 0.043 & 1.656 & 1.818 & 94.7 & 0.519 & 2.3 & 0.27 & 0.03 & -0.24 & -0.465 & 4.9 \\
\hline N2 & 0.076 & 1.655 & 1.820 & 98.8 & 0.506 & 5.3 & 0.32 & 0.02 & -0.30 & -0.460 & 6.1 \\
\hline N3 & 0.073 & 1.669 & 1.817 & 102.3 & 0.499 & 8.0 & 0.34 & 0.02 & -0.32 & -0.464 & 6.6 \\
\hline N4 & 0.077 & 1.667 & 1.819 & 99.7 & 0.500 & 3.4 & 0.34 & 0.02 & -0.32 & -0.468 & 6.1 \\
\hline N5 & 0.077 & 1.658 & 1.819 & 100.1 & 0.511 & 3.6 & 0.32 & 0.02 & -0.30 & -0.457 & 4.8 \\
\hline As1 & 0.072 & 1.607 & 1.824 & 94.5 & 0.544 & 3.3 & 0.31 & 0.07 & -0.24 & -0.436 & 5.1 \\
\hline As2 & 0.061 & 1.627 & 1.824 & 96.3 & 0.520 & 2.2 & 0.45 & 0.05 & -0.39 & -0.450 & 6.4 \\
\hline $\mathrm{O} 1$ & 0.062 & 1.664 & 1.816 & 93.6 & 0.512 & 1.5 & 0.18 & 0.01 & -0.17 & -0.453 & 3.4 \\
\hline $\mathrm{O} 2$ & 0.093 & 1.654 & 1.820 & 96.5 & 0.524 & 1.7 & 0.19 & 0.01 & -0.18 & -0.447 & 6.4 \\
\hline $\mathrm{O} 3$ & 0.089 & 1.659 & 1.818 & 95.9 & 0.520 & 2.2 & 0.21 & 0.01 & -0.20 & -0.446 & 6.4 \\
\hline $\mathrm{O} 4$ & 0.087 & 1.658 & 1.819 & 96.7 & 0.521 & 1.5 & 0.23 & 0.02 & -0.22 & -0.459 & 7,1 \\
\hline $\mathrm{H} 1$ & 0.063 & 1.644 & 1.822 & 94.9 & 0.517 & 4.7 & 0.34 & 0.09 & -0.25 & -0.460 & 7.0 \\
\hline $\mathrm{H} 2$ & 0.076 & 1.654 & 1.819 & 94.3 & 0.506 & 2.4 & 0.31 & 0.07 & -0.23 & -0.457 & 6.0 \\
\hline $\mathrm{H} 3$ & 0.077 & 1.656 & 1.820 & 94.2 & 0.510 & 2.6 & 0.36 & 0.06 & -0.30 & -0.471 & 8.3 \\
\hline $\mathrm{H} 4$ & 0.075 & 1.658 & 1.819 & 94.3 & 0.509 & 2.5 & 0.36 & 0.06 & -0.30 & -0.477 & 9.0 \\
\hline S1 & 0.079 & 1.630 & 1.821 & 93.6 & 0.537 & 2.1 & 0.29 & 0.04 & -0.25 & -0.434 & 4.0 \\
\hline S2 & 0.075 & 1.636 & 1.822 & 92.4 & 0.521 & 3.2 & 0.31 & 0.04 & -0.26 & -0.448 & 5.8 \\
\hline S3 & 0.076 & 1.622 & 1.821 & 96.8 & 0.528 & 2.4 & 0.33 & 0.05 & -0.28 & -0.450 & 7.9 \\
\hline S4 & 0.070 & 1.649 & 1.819 & 95.1 & 0.506 & 2.8 & 0.30 & 0.04 & -0.26 & -0.436 & 10.1 \\
\hline S5 & 0.076 & 1.608 & 1.827 & 92.7 & 0.530 & 3.5 & 0.27 & 0.08 & -0.20 & -0.426 & 4.0 \\
\hline $\mathrm{C} 1$ & 0.031 & 1.552 & 1.827 & 98.3 & 0.533 & 9.1 & 0.60 & 0.45 & -0.15 & -0.409 & 4.9 \\
\hline C2 & 0.036 & 1.552 & 1.824 & 99.1 & 0.522 & 9.8 & 0.66 & 0.49 & -0.17 & -0.392 & 4.0 \\
\hline CN1 & 0.085 & 1.621 & 1.823 & 94.5 & 0.544 & 2.4 & 0.24 & 0.17 & -0.07 & -0.441 & 7.0 \\
\hline $\mathrm{NC1}$ & 0.054 & 1.579 & 1.829 & 94.7 & 0.544 & 2.3 & 0.39 & 0.27 & -0.13 & -0.425 & 7.1 \\
\hline NC2 & 0.069 & 1.586 & 1.828 & 94.4 & 0.542 & 2.6 & 0.37 & 0.24 & -0.13 & -0.429 & 6.5 \\
\hline $\mathrm{CO}$ & 0.066 & 1.536 & 1.835 & 94.3 & 0.567 & 2.3 & 0.32 & 0.35 & 0.03 & -0.362 & 3.5 \\
\hline CS & 0.046 & 1.516 & 1.836 & 95.2 & 0.555 & 4.5 & 0.38 & 0.48 & 0.09 & -0.369 & 3.6 \\
\hline $\mathrm{CSe}$ & 0.042 & 1.510 & 1.836 & 95.5 & 0.554 & 5.2 & 0.38 & 0.50 & 0.12 & -0.365 & 3.7 \\
\hline
\end{tabular}

${ }^{a}$ Molecular descriptors (md). md50: Min electroph. react. index for a Ru atom, md82: Max bond order of a Ru atom, $\mathrm{md90:} \mathrm{Ru}=\mathrm{CH}_{2}$ bond length, md92: $\mathrm{L}-\mathrm{Ru}=\mathrm{C}$ angle, md96: Wiberg bond index: average of the bonds $\mathrm{Ru}-\mathrm{Cl}_{\mathrm{a}}$ and $\mathrm{Ru}-\mathrm{Cl}_{\mathrm{b}}, \mathrm{md} 103$ : Steric exchange energy between

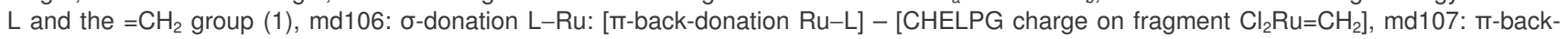
donation $\mathrm{Ru}-\mathrm{L}$, md113: $\mathrm{CHELPG}$ charge on the group $\mathrm{Cl}_{2} \mathrm{Ru}=\mathrm{CH}_{2}$, md116: $\mathrm{CHELPG}$ charge average on the $\mathrm{Cl}$ belonging to the $\mathrm{Cl}_{2} \mathrm{Ru}=\mathrm{CH}_{2}$ group, md117: Total dipole moment (CHELPG). ${ }^{\circ}$ The structures of ligands $L$ are provided in Chart 2 of the paper. ${ }^{\circ}$ The structures of these ligands are provided in Chart 3 of the paper. 
Table S3. Predicted (PLS model) and explicitly calculated (DFT) productivities for $77 \mathrm{Ru}$-complexes of ligands (L). ${ }^{\mathrm{a}}$

\begin{tabular}{|c|c|c|c|c|c|c|c|c|}
\hline \multirow[b]{2}{*}{ L } & \multicolumn{2}{|c|}{ productivity } & \multirow[b]{2}{*}{$\mathrm{L}$} & \multicolumn{2}{|c|}{ productivity } & \multirow[b]{2}{*}{ L } & \multicolumn{2}{|c|}{ productivity } \\
\hline & predicted & calculated & & predicted & calculated & & predicted & calculated \\
\hline A1 & -6.4 & -6.5 & A28 & -4.0 & -4.3 & N2 & -4.3 & -4.6 \\
\hline A2 & -2.8 & -2.8 & A29 & -2.3 & -1.6 & N3 & -0.4 & \\
\hline A3 & 1.0 & 1.5 & A31 & -2.6 & & N4 & -0.7 & 0.0 \\
\hline A4 & 0.0 & & A32 & -4.5 & & N5 & -5.6 & \\
\hline A5 & 3.0 & 2.8 & A33 & -5.0 & & As1 & -12.8 & -12.3 \\
\hline A6 & 1.5 & 0.9 & A34 & -6.0 & & As2 & -9.0 & -8.9 \\
\hline A8 & 5.3 & 5.6 & A35 & -1.1 & -0.5 & $\mathrm{O} 2$ & -5.5 & \\
\hline A9 & 2.1 & & A37 & -1.6 & -1.1 & $\mathrm{O} 3$ & -3.9 & \\
\hline A10 & 1.8 & 2.3 & A38 & -1.9 & & $\mathrm{O} 4$ & -3.1 & -3.1 \\
\hline A11 & -1.4 & -0.8 & P1 & -11.7 & -12.9 & $\mathrm{H} 1$ & -4.4 & \\
\hline A12 & 2.4 & 2.6 & P2 & -7.3 & & $\mathrm{H} 2$ & -3.5 & \\
\hline A13 & 2.3 & 1.8 & P3 & -5.4 & & H3 & -1.9 & -2.5 \\
\hline A14 & 1.1 & 1.2 & P4 & -5.5 & -5.2 & $\mathrm{H} 4$ & -1.1 & \\
\hline A15 & 2.3 & 1.7 & P5 & -4.4 & & S1 & -9.8 & \\
\hline A16 & 2.5 & 2.8 & P6 & -3.2 & & S2 & -5.1 & -5.6 \\
\hline A17 & 3.8 & & P7 & -3.5 & -2.9 & S3 & -9.7 & -9.9 \\
\hline A18 & 0.3 & & P8 & -10.7 & -11.3 & S4 & -3.3 & \\
\hline A19 & 0.4 & & P9 & -9.5 & & S5 & -14.6 & -14.3 \\
\hline A20 & 4.1 & 3.7 & P10 & -7.9 & & C1 & -9.2 & \\
\hline A21 & 2.5 & & P11 & -10.7 & & NC1 & -15.2 & -15.2 \\
\hline A22 & 2.1 & & P12 & -7.9 & & NC2 & -12.8 & \\
\hline A23 & 1.1 & 0.2 & P13 & -14.9 & -14.8 & $\mathrm{CN} 1$ & -12.6 & \\
\hline A24 & 1.8 & 2.5 & P14 & -14.4 & & $\mathrm{CO}$ & -23.7 & -23.8 \\
\hline A25 & 1.4 & 1.4 & P15 & -16.0 & & CS & -23.5 & \\
\hline A26 & 0.3 & 0.4 & P16 & -21.9 & -21.9 & $\mathrm{CSe}$ & -22.2 & -21.8 \\
\hline A27 & 0.0 & -0.9 & $\mathrm{~N} 1$ & -4.7 & -5.3 & & & \\
\hline
\end{tabular}


Dendrogram Using Mahalanobis Distance on 8 PCs

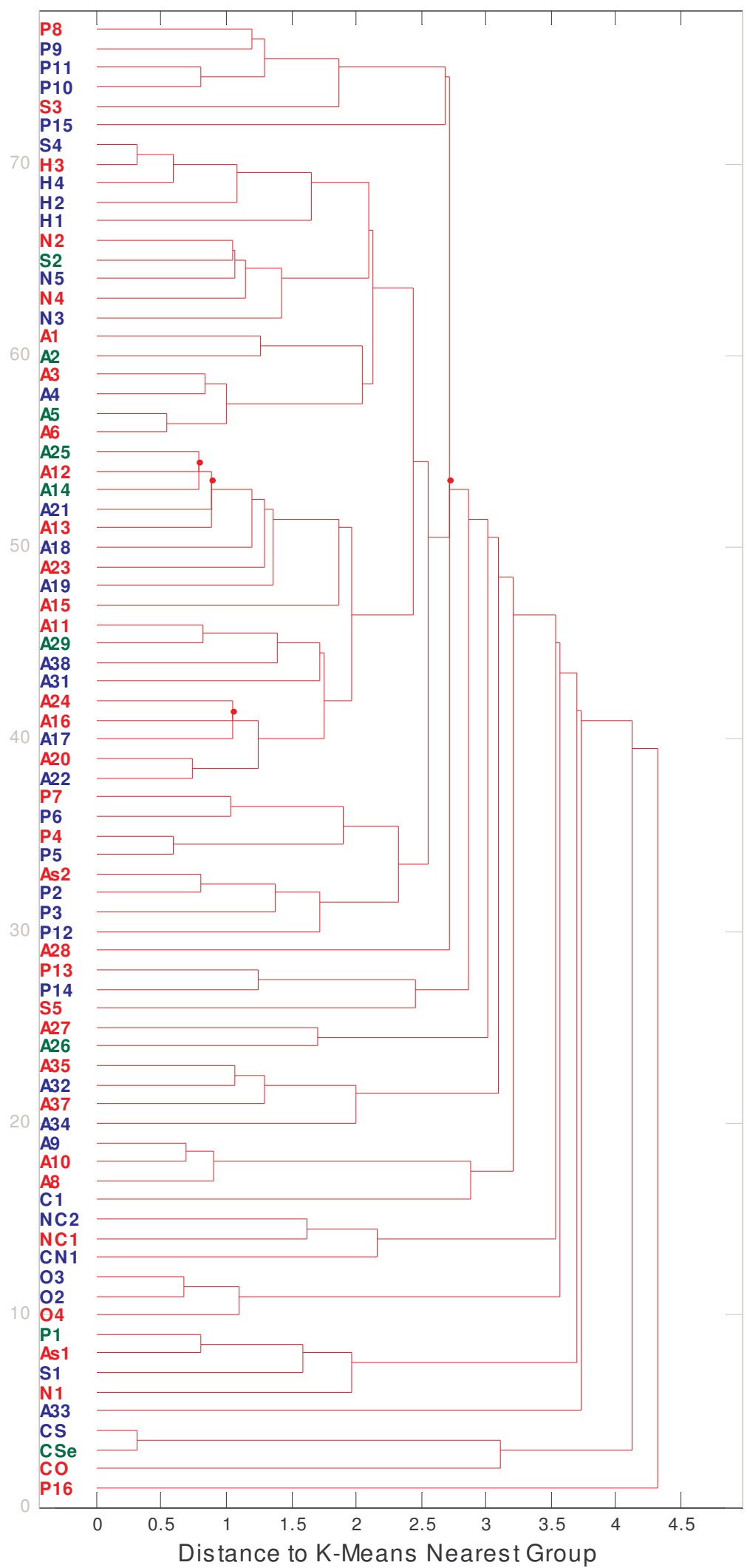

Figure S1. Dendrogram showing the cluster analysis with eight principal components (PCs) using the Mahalanobis distance. Color-coding: Calibration set, red; prediction set, blue; test set, green. See Chart 2 in the main publication for ligand structures. 
Additional results from the $\mathrm{PCA}$ for $\mathrm{X}_{77 \times 122}\left(77 \mathrm{LCl}_{2} \mathrm{Ru}=\mathrm{CH}_{2}\right.$ complexes and 122 molecular descriptors)

Table S4. Percent of variance captured by PCA model

\begin{tabular}{cccc}
\hline $\begin{array}{c}\text { Principal } \\
\text { Component }\end{array}$ & $\begin{array}{c}\text { Eigenvalue } \\
\text { of } \operatorname{Cov}(\mathbf{X})\end{array}$ & \% Variance & $\begin{array}{c}\text { \% Variance } \\
\text { Cumulative }\end{array}$ \\
\hline 1 & 47.88 & 39.24 & 39.24 \\
2 & 17.14 & 14.05 & 53.30 \\
3 & 9.36 & 7.67 & 60.97 \\
4 & 6.52 & 5.34 & 66.31 \\
5 & 4.21 & 3.45 & 69.76 \\
6 & 3.91 & 3.21 & 72.97 \\
7 & 3.69 & 3.02 & 75.99 \\
8 & 3.39 & 2.78 & 78.77 \\
9 & 2.50 & 2.05 & 80.81 \\
10 & 2.43 & 1.99 & 82.80 \\
11 & 2.03 & 1.66 & 84.47 \\
12 & 1.92 & 1.57 & 86.04 \\
\hline
\end{tabular}

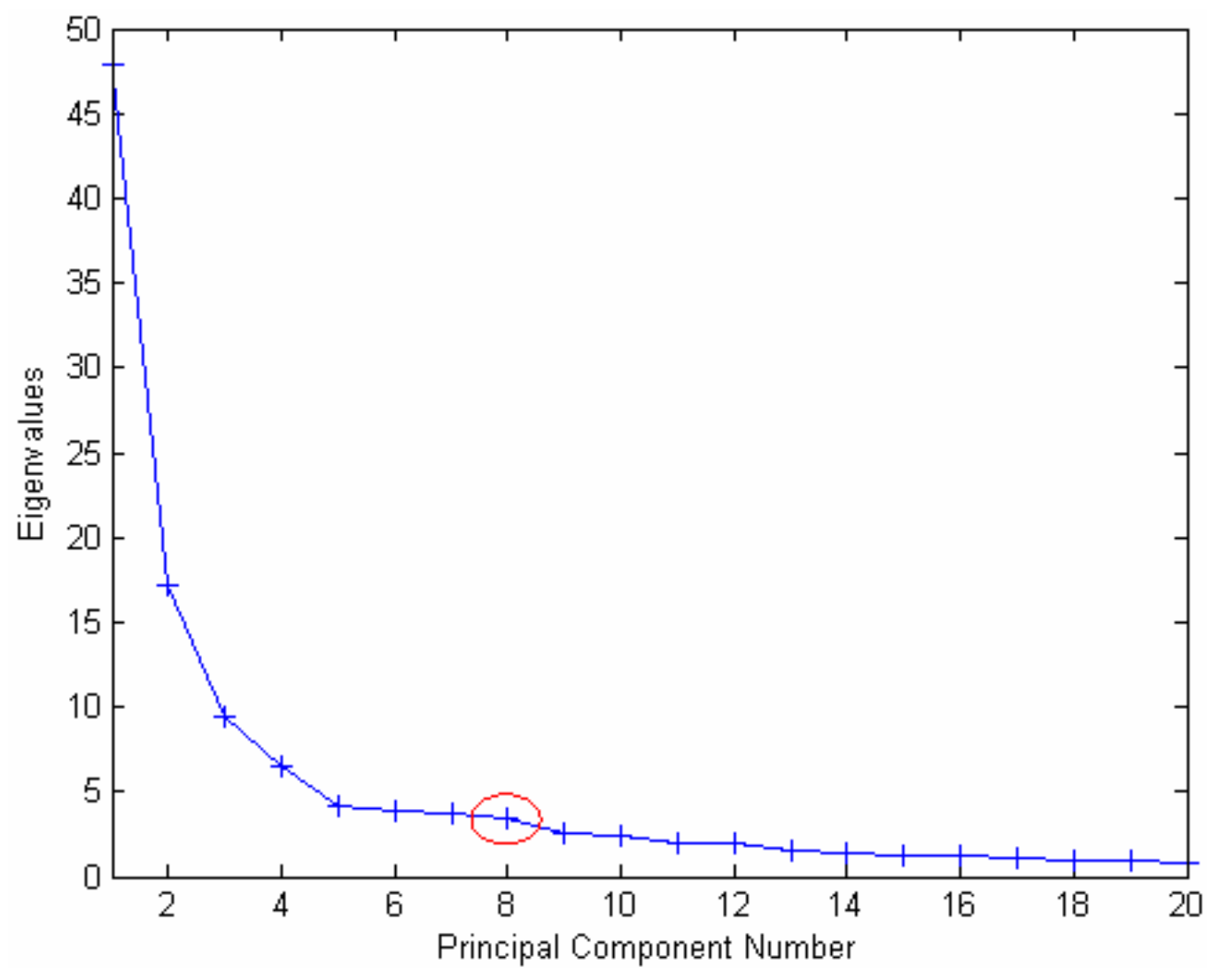

Figure S2. Eigenvalues plot for $\boldsymbol{X}_{77 \times 122}$ 
Score plots - PCA for $\mathbf{X}_{77 \times 122}$

2D Score plot $t_{1}$ vs $t_{2}$

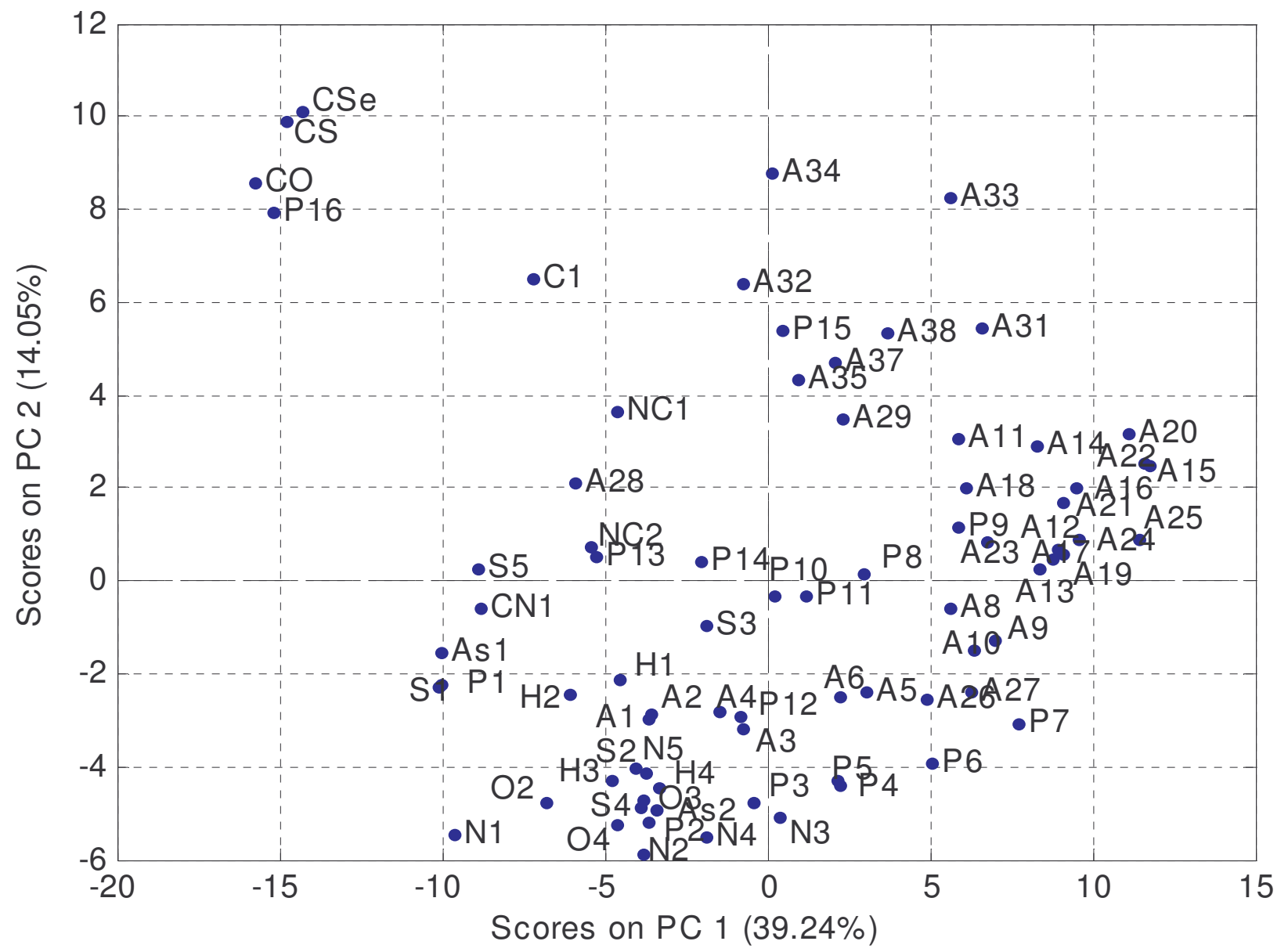


2D Score plot $t_{1} v_{s} t_{3}$

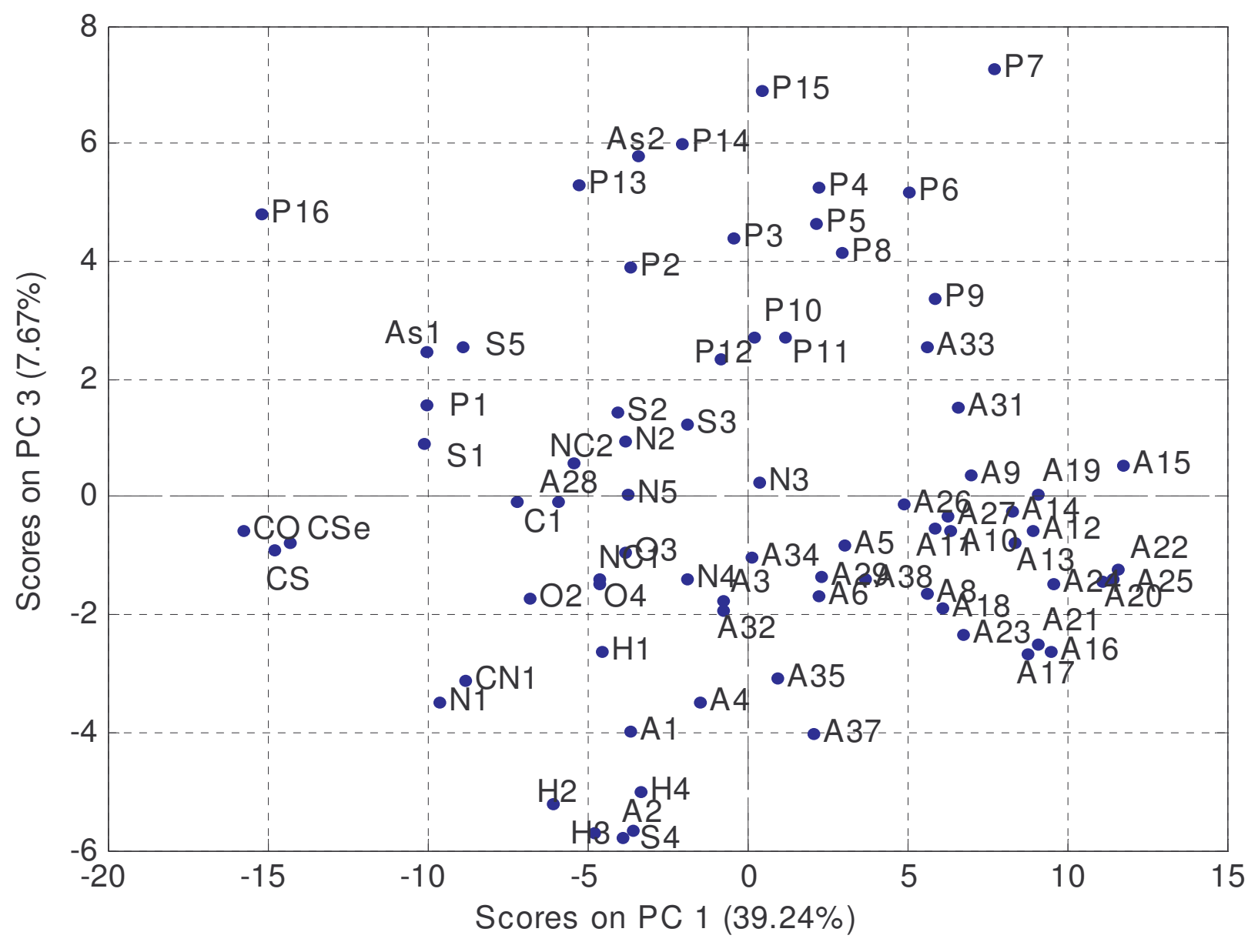


2D Score plot $t_{1} v_{s} t_{4}$

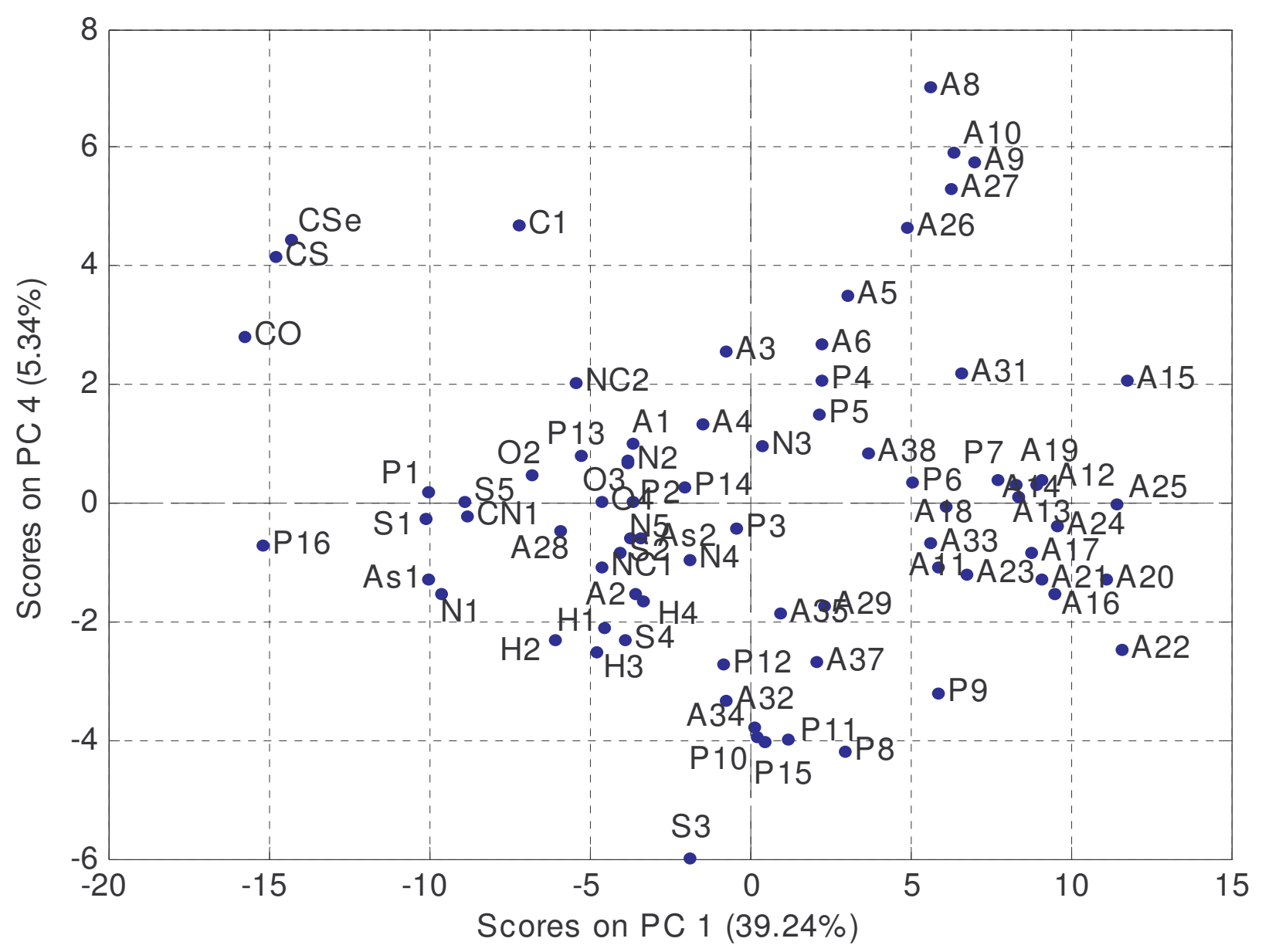


2D Score plot $t_{1} v_{s} t_{5}$

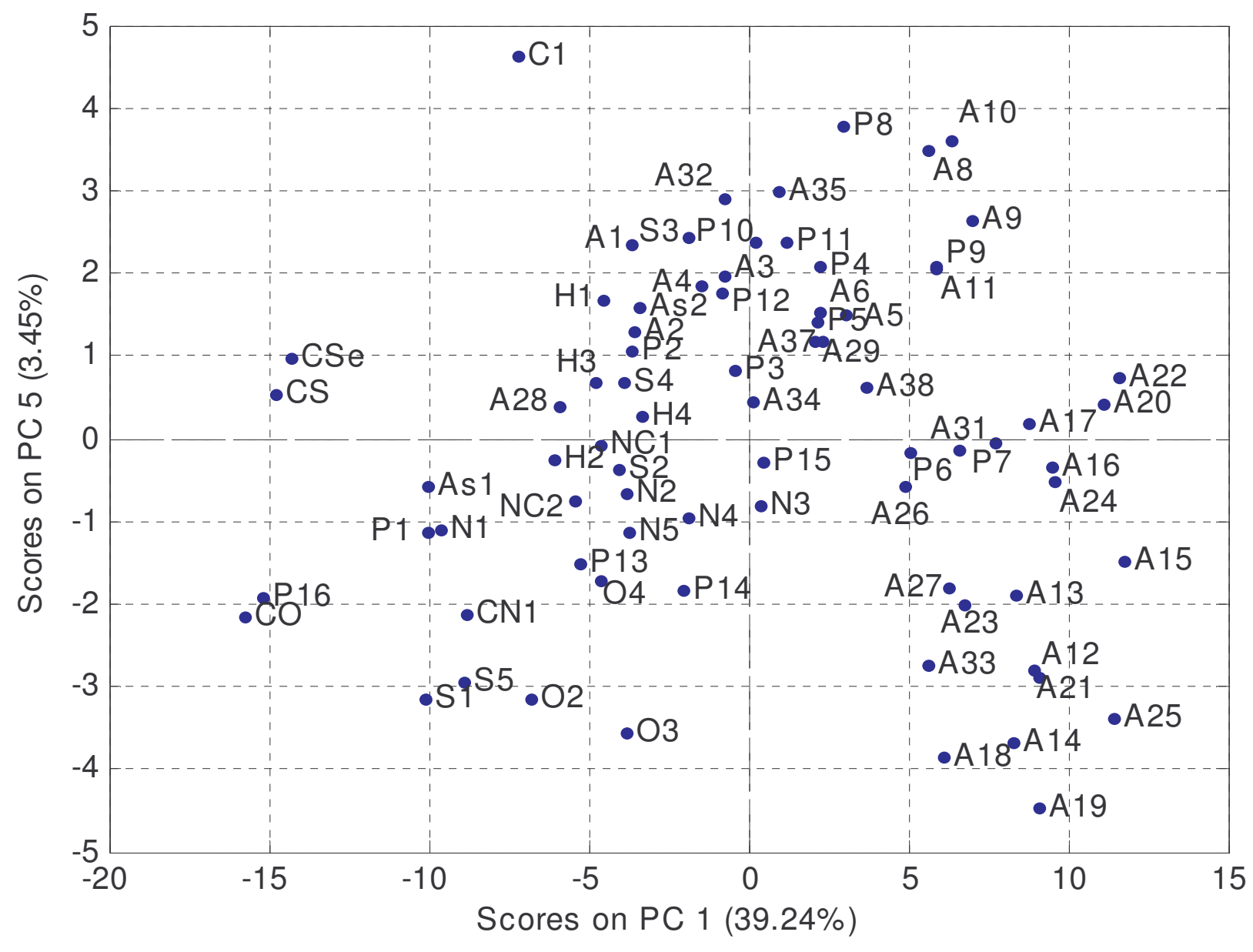


2D Score plot $t_{1} v_{s} t_{6}$

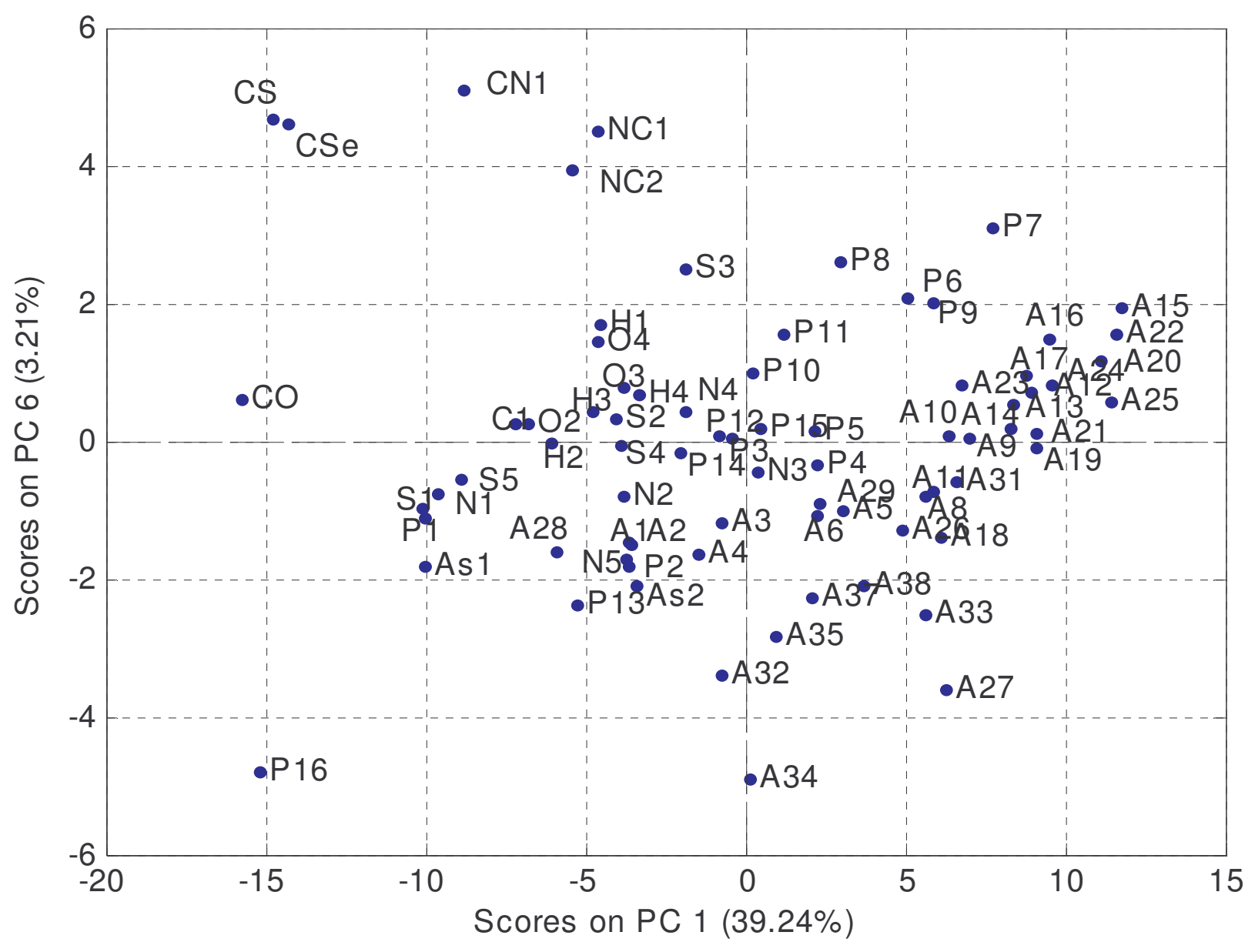


2D Score plot $t_{3} v s t_{2}$

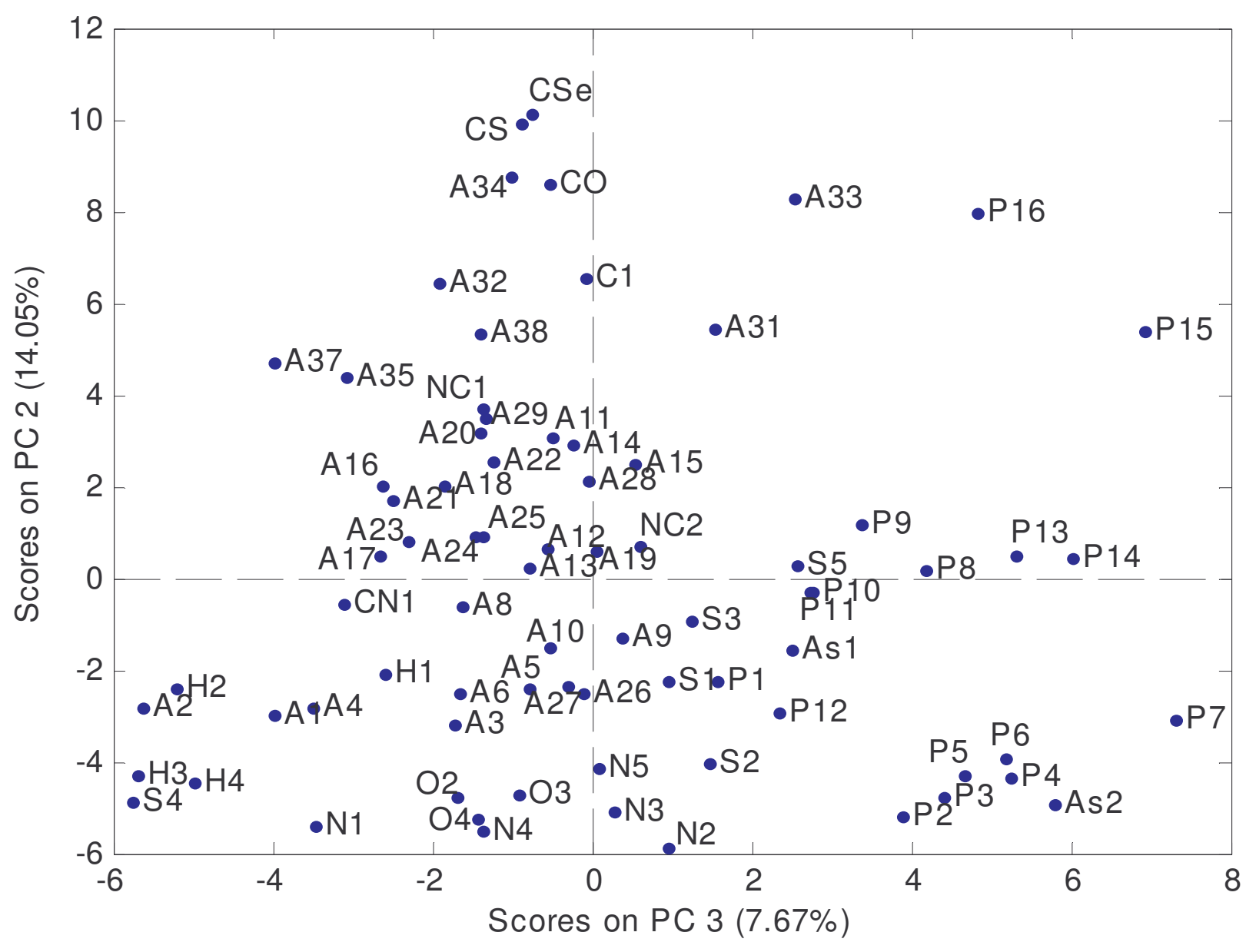


3D Score plot $t_{1} v_{s} t_{2} v s t_{3}$

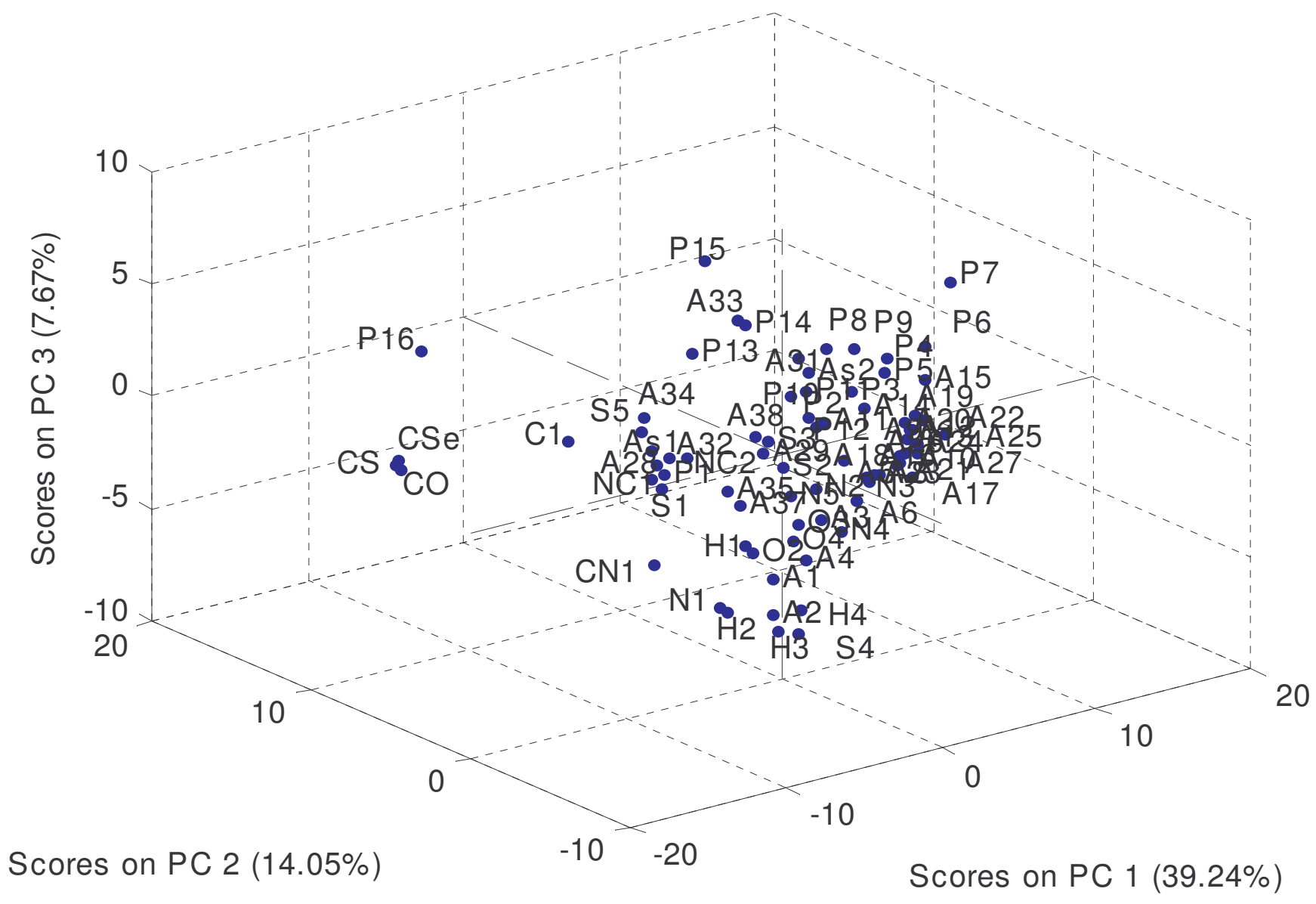


Table S5. Score values achieved from the principal component analysis (PCA) of $\mathbf{X}_{77 \times 122}$

\begin{tabular}{|c|c|c|c|c|c|c|c|c|c|c|}
\hline \multirow[b]{2}{*}{$\mathrm{L}^{\mathrm{a}}$} & \multicolumn{10}{|c|}{ Scores from principal component analysis of the molecular descriptor matrix $\boldsymbol{X}_{77 \times 122}$} \\
\hline & $t_{1}$ & $t_{2}$ & $t_{3}$ & $t_{4}$ & $t_{5}$ & $t_{6}$ & $t_{7}$ & $t_{8}$ & $t_{9}$ & $\boldsymbol{t}_{10}$ \\
\hline A1 & -3.68 & -2.97 & -3.98 & 1.03 & 2.35 & -1.46 & 3.32 & -0.30 & -1.41 & -1.07 \\
\hline A2 & -3.58 & -2.83 & -5.64 & -1.53 & 1.30 & -1.48 & 3.54 & -0.30 & -0.14 & -0.53 \\
\hline A3 & -0.75 & -3.17 & -1.74 & 2.57 & 1.99 & -1.17 & 1.47 & 0.05 & -0.62 & 0.29 \\
\hline A4 & -1.47 & -2.82 & -3.49 & 1.33 & 1.86 & -1.62 & 1.07 & -0.10 & -0.63 & 0.22 \\
\hline A5 & 2.99 & -2.37 & -0.80 & 3.54 & 1.51 & -1.02 & 0.79 & -0.34 & -1.61 & 0.95 \\
\hline A6 & 2.25 & -2.49 & -1.66 & 2.72 & 1.54 & -1.07 & 0.38 & -0.78 & -1.48 & 1.28 \\
\hline A8 & 5.61 & -0.59 & -1.64 & 7.02 & 3.48 & -0.77 & -2.01 & 0.17 & -0.17 & -0.01 \\
\hline A9 & 6.94 & -1.28 & 0.37 & 5.75 & 2.63 & 0.06 & -1.12 & 0.07 & 1.63 & -1.84 \\
\hline $\mathrm{A} 10$ & 6.34 & -1.49 & -0.54 & 5.94 & 3.62 & 0.09 & -1.84 & 0.00 & 1.71 & -3.05 \\
\hline A11 & 5.82 & 3.06 & -0.51 & -1.05 & 2.06 & -0.73 & -1.05 & 0.84 & 0.59 & -2.08 \\
\hline $\mathrm{A} 12$ & 8.91 & 0.67 & -0.58 & 0.33 & -2.81 & 0.72 & 1.54 & 0.66 & -0.51 & 1.74 \\
\hline $\mathrm{A} 13$ & 8.33 & 0.25 & -0.78 & 0.10 & -1.89 & 0.54 & 0.44 & 1.44 & -0.76 & 0.12 \\
\hline A14 & 8.25 & 2.94 & -0.25 & 0.33 & -3.67 & 0.18 & 0.75 & 0.63 & -1.56 & 0.21 \\
\hline A15 & 11.72 & 2.50 & 0.55 & 2.09 & -1.47 & 1.93 & -0.13 & 0.84 & 2.08 & 2.41 \\
\hline $\mathrm{A} 16$ & 9.50 & 2.02 & -2.63 & -1.52 & -0.33 & 1.48 & 1.26 & 0.77 & 0.02 & 1.69 \\
\hline A17 & 8.76 & 0.48 & -2.66 & -0.84 & 0.19 & 0.97 & -0.27 & 1.73 & -0.88 & -2.26 \\
\hline A18 & 6.07 & 2.02 & -1.85 & -0.04 & -3.83 & -1.38 & 1.48 & 0.83 & -1.07 & -0.52 \\
\hline A19 & 9.08 & 0.59 & 0.06 & 0.42 & -4.46 & -0.08 & 2.59 & -0.46 & -0.90 & -0.01 \\
\hline A20 & 11.07 & 3.18 & -1.41 & -1.26 & 0.41 & 1.18 & -0.85 & 1.41 & 1.93 & -2.42 \\
\hline A21 & 9.04 & 1.71 & -2.50 & -1.28 & -2.89 & 0.14 & 1.24 & 0.62 & -0.57 & 2.02 \\
\hline A22 & 11.61 & 2.57 & -1.24 & -2.46 & 0.76 & 1.56 & -1.53 & 2.03 & 1.60 & -2.27 \\
\hline A23 & 6.71 & 0.83 & -2.31 & -1.17 & -2.01 & 0.83 & 2.38 & -0.21 & -1.38 & 1.10 \\
\hline A24 & 9.56 & 0.93 & -1.45 & -0.37 & -0.52 & 0.84 & 0.08 & -0.02 & -0.80 & 1.82 \\
\hline A25 & 11.39 & 0.90 & -1.37 & 0.00 & -3.39 & 0.58 & 0.48 & 0.95 & -0.59 & 0.87 \\
\hline A26 & 4.88 & -2.52 & -0.11 & 4.65 & -0.58 & -1.28 & 0.84 & -2.24 & 0.78 & 2.38 \\
\hline A27 & 6.28 & -2.36 & -0.30 & 5.31 & -1.81 & -3.60 & -1.10 & -2.62 & 1.98 & 0.76 \\
\hline A28 & -5.91 & 2.12 & -0.06 & -0.45 & 0.39 & -1.59 & -3.73 & 0.55 & -6.71 & 2.81 \\
\hline A29 & 2.30 & 3.50 & -1.33 & -1.71 & 1.19 & -0.88 & -0.71 & 0.47 & -2.22 & -2.15 \\
\hline A31 & 6.55 & 5.46 & 1.54 & 2.21 & -0.13 & -0.57 & -0.61 & 1.49 & 0.01 & -3.58 \\
\hline A32 & -0.79 & 6.44 & -1.91 & -3.32 & 2.91 & -3.39 & -0.72 & -2.72 & 4.36 & 2.54 \\
\hline A33 & 5.58 & 8.28 & 2.54 & -0.67 & -2.74 & -2.49 & -3.86 & 1.24 & -0.86 & -1.00 \\
\hline A34 & 0.10 & 8.77 & -1.03 & -3.78 & 0.47 & -4.88 & 1.45 & -1.59 & 1.47 & 1.36 \\
\hline A35 & 0.94 & 4.37 & -3.08 & -1.85 & 2.99 & -2.83 & 0.01 & -2.06 & 1.95 & 0.84 \\
\hline A37 & 2.08 & 4.71 & -4.00 & -2.67 & 1.18 & -2.25 & 0.74 & -1.93 & 1.96 & 3.00 \\
\hline A38 & 3.66 & 5.36 & -1.40 & 0.87 & 0.63 & -2.09 & -0.30 & 1.37 & -0.34 & -3.80 \\
\hline
\end{tabular}


Continuation of Table S5.

\begin{tabular}{|c|c|c|c|c|c|c|c|c|c|c|}
\hline \multirow[b]{2}{*}{$\mathrm{L}$} & \multicolumn{10}{|c|}{ Scores from principal component analysis of the molecular descriptor matrix $\boldsymbol{X}_{77 \times 122}$} \\
\hline & $t_{1}$ & $t_{2}$ & $t_{3}$ & $t_{4}$ & $t_{5}$ & $\boldsymbol{t}_{6}$ & $t_{7}$ & $\boldsymbol{t}_{8}$ & $t_{9}$ & $\boldsymbol{t}_{10}$ \\
\hline P1 & -10.00 & -2.23 & 1.57 & 0.20 & -1.13 & -1.12 & 0.74 & 5.80 & 1.79 & 1.87 \\
\hline P2 & -3.65 & -5.16 & 3.90 & 0.03 & 1.06 & -1.80 & 3.53 & -0.05 & 0.31 & -0.31 \\
\hline P3 & -0.43 & -4.78 & 4.41 & -0.42 & 0.84 & 0.04 & 2.37 & -0.24 & -0.58 & -0.33 \\
\hline P4 & 2.20 & -4.37 & 5.26 & 2.08 & 2.08 & -0.32 & -0.40 & 0.12 & 0.33 & 1.05 \\
\hline P5 & 2.14 & -4.30 & 4.67 & 1.51 & 1.41 & 0.15 & 0.19 & 0.34 & 0.36 & 0.18 \\
\hline P6 & 5.07 & -3.92 & 5.19 & 0.35 & -0.17 & 2.10 & 0.40 & 0.21 & 0.65 & 0.45 \\
\hline P7 & 7.68 & -3.05 & 7.30 & 0.40 & -0.03 & 3.11 & 0.08 & 0.87 & 0.84 & 1.02 \\
\hline P8 & 2.92 & 0.19 & 4.17 & -4.20 & 3.80 & 2.63 & -1.45 & -0.45 & 1.28 & 1.11 \\
\hline P9 & 5.82 & 1.20 & 3.36 & -3.18 & 2.08 & 2.03 & -1.64 & 0.27 & 0.13 & 1.66 \\
\hline P10 & 0.20 & -0.30 & 2.74 & -3.93 & 2.37 & 0.98 & 0.24 & 0.72 & -0.13 & -0.10 \\
\hline P11 & 1.13 & -0.31 & 2.72 & -3.96 & 2.39 & 1.56 & 0.22 & -0.64 & -0.20 & -0.80 \\
\hline P12 & -0.86 & -2.92 & 2.35 & -2.70 & 1.77 & 0.08 & 2.16 & 0.86 & -0.70 & -0.14 \\
\hline P13 & -5.26 & 0.52 & 5.31 & 0.82 & -1.50 & -2.37 & 1.62 & -2.63 & -1.28 & -1.42 \\
\hline P14 & -2.06 & 0.42 & 6.00 & 0.30 & -1.82 & -0.17 & 1.77 & -2.69 & -1.66 & -1.03 \\
\hline P15 & 0.44 & 5.41 & 6.93 & -4.03 & -0.28 & 0.19 & -1.27 & -1.91 & -1.01 & -1.81 \\
\hline P16 & -15.18 & 7.96 & 4.83 & -0.68 & -1.93 & -4.78 & -0.96 & -0.65 & -0.51 & -0.52 \\
\hline $\mathrm{N} 1$ & -9.62 & -5.42 & -3.47 & -1.50 & -1.10 & -0.76 & 0.36 & 5.15 & 3.11 & -2.12 \\
\hline N2 & -3.86 & -5.88 & 0.94 & 0.73 & -0.65 & -0.78 & -1.36 & 0.35 & 1.35 & 0.68 \\
\hline N3 & 0.38 & -5.08 & 0.27 & 0.99 & -0.81 & -0.44 & -2.58 & -0.98 & -0.08 & 0.64 \\
\hline N4 & -1.89 & -5.50 & -1.36 & -0.96 & -0.96 & 0.44 & -0.01 & 0.52 & -0.76 & -1.65 \\
\hline N5 & -3.76 & -4.12 & 0.07 & -0.56 & -1.13 & -1.69 & -0.33 & -0.50 & -1.00 & -1.87 \\
\hline As1 & -10.08 & -1.54 & 2.49 & -1.27 & -0.56 & -1.81 & 0.63 & 5.62 & 2.04 & 2.22 \\
\hline As2 & -3.40 & -4.94 & 5.80 & -0.56 & 1.58 & -2.07 & 4.16 & 0.09 & 0.32 & 0.03 \\
\hline $\mathrm{O} 2$ & -6.82 & -4.74 & -1.69 & 0.47 & -3.15 & 0.27 & -5.03 & -2.18 & 2.33 & 1.12 \\
\hline O3 & -3.85 & -4.72 & -0.93 & 0.68 & -3.55 & 0.78 & -4.93 & -2.74 & 1.28 & 1.19 \\
\hline O4 & -4.67 & -5.23 & -1.45 & 0.03 & -1.71 & 1.46 & -4.06 & -2.07 & 0.42 & -0.38 \\
\hline $\mathrm{H} 1$ & -4.57 & -2.09 & -2.60 & -2.10 & 1.68 & 1.70 & -1.80 & 0.28 & -0.01 & 0.33 \\
\hline H2 & -6.10 & -2.41 & -5.20 & -2.28 & -0.24 & 0.00 & -1.00 & 0.50 & -0.57 & -1.48 \\
\hline $\mathrm{H} 3$ & -4.77 & -4.28 & -5.71 & -2.49 & 0.69 & 0.44 & -0.17 & -0.51 & -1.42 & -0.92 \\
\hline $\mathrm{H} 4$ & -3.39 & -4.43 & -4.98 & -1.64 & 0.26 & 0.70 & 0.18 & -0.90 & -0.62 & 0.25 \\
\hline S1 & -10.10 & -2.24 & 0.94 & -0.25 & -3.15 & -0.97 & -0.97 & 4.71 & 0.14 & 0.65 \\
\hline S2 & -4.05 & -4.03 & 1.45 & -0.81 & -0.37 & 0.33 & -0.60 & 0.11 & -1.44 & 0.79 \\
\hline S3 & -1.92 & -0.93 & 1.25 & -5.98 & 2.45 & 2.50 & -3.07 & -0.78 & -0.41 & 0.34 \\
\hline S4 & -3.94 & -4.84 & -5.76 & -2.31 & 0.68 & -0.05 & -0.16 & -0.57 & -1.90 & -0.57 \\
\hline S5 & -8.89 & 0.28 & 2.56 & 0.03 & -2.93 & -0.55 & -2.06 & -3.18 & -0.38 & -2.97 \\
\hline C1 & -7.19 & 6.53 & -0.07 & 4.69 & 4.64 & 0.26 & -1.70 & 0.87 & -3.54 & 2.15 \\
\hline CN1 & -8.82 & -0.57 & -3.11 & -0.20 & -2.11 & 5.11 & 1.04 & -2.36 & 1.28 & -0.86 \\
\hline $\mathrm{NC} 1$ & -4.64 & 3.69 & -1.38 & -1.07 & -0.06 & 4.51 & 3.64 & -3.38 & 0.18 & -0.16 \\
\hline NC2 & -5.45 & 0.72 & 0.60 & 2.05 & -0.73 & 3.95 & 3.53 & -3.63 & 0.91 & -0.90 \\
\hline $\mathrm{CO}$ & -15.78 & 8.60 & -0.55 & 2.84 & -2.15 & 0.61 & 2.31 & -0.61 & 3.25 & -1.31 \\
\hline CS & -14.34 & 10.11 & -0.76 & 4.46 & 0.97 & 4.60 & 0.06 & 1.82 & -0.80 & 1.57 \\
\hline $\mathrm{CSe}$ & -14.80 & 9.92 & -0.89 & 4.16 & 0.53 & 4.70 & 0.34 & 1.95 & -0.14 & 0.51 \\
\hline
\end{tabular}

${ }^{a}$ The chemical structures of the ligands, $L$, are provided in Chart 2 of the paper. 
Loadings plots - PCA of $\mathrm{X}_{77 \times 122}$

2D Loadings plot $p_{1}$ vs $p_{2}$

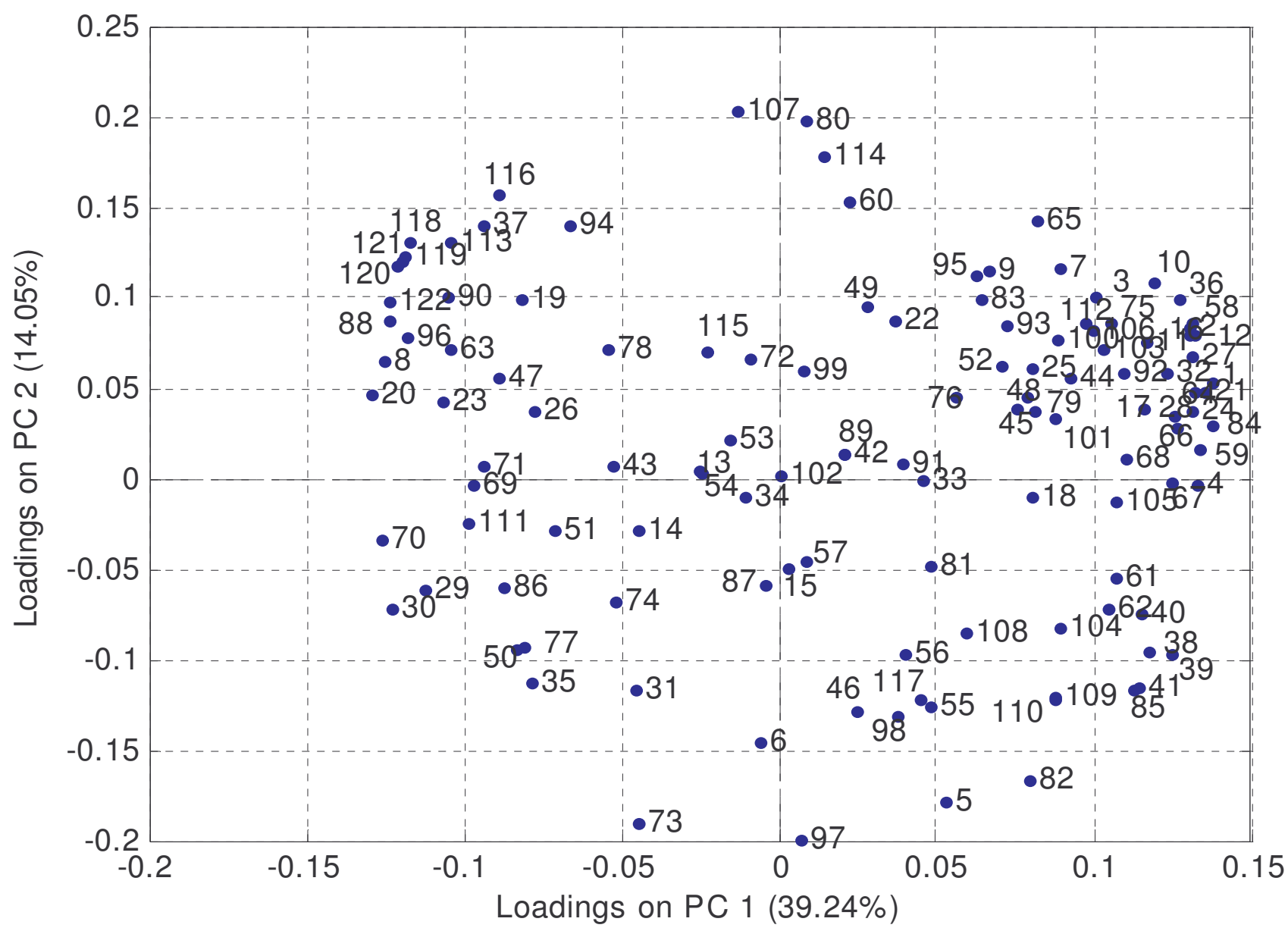


2D Loadings plot $p_{1}$ vs $p_{3}$

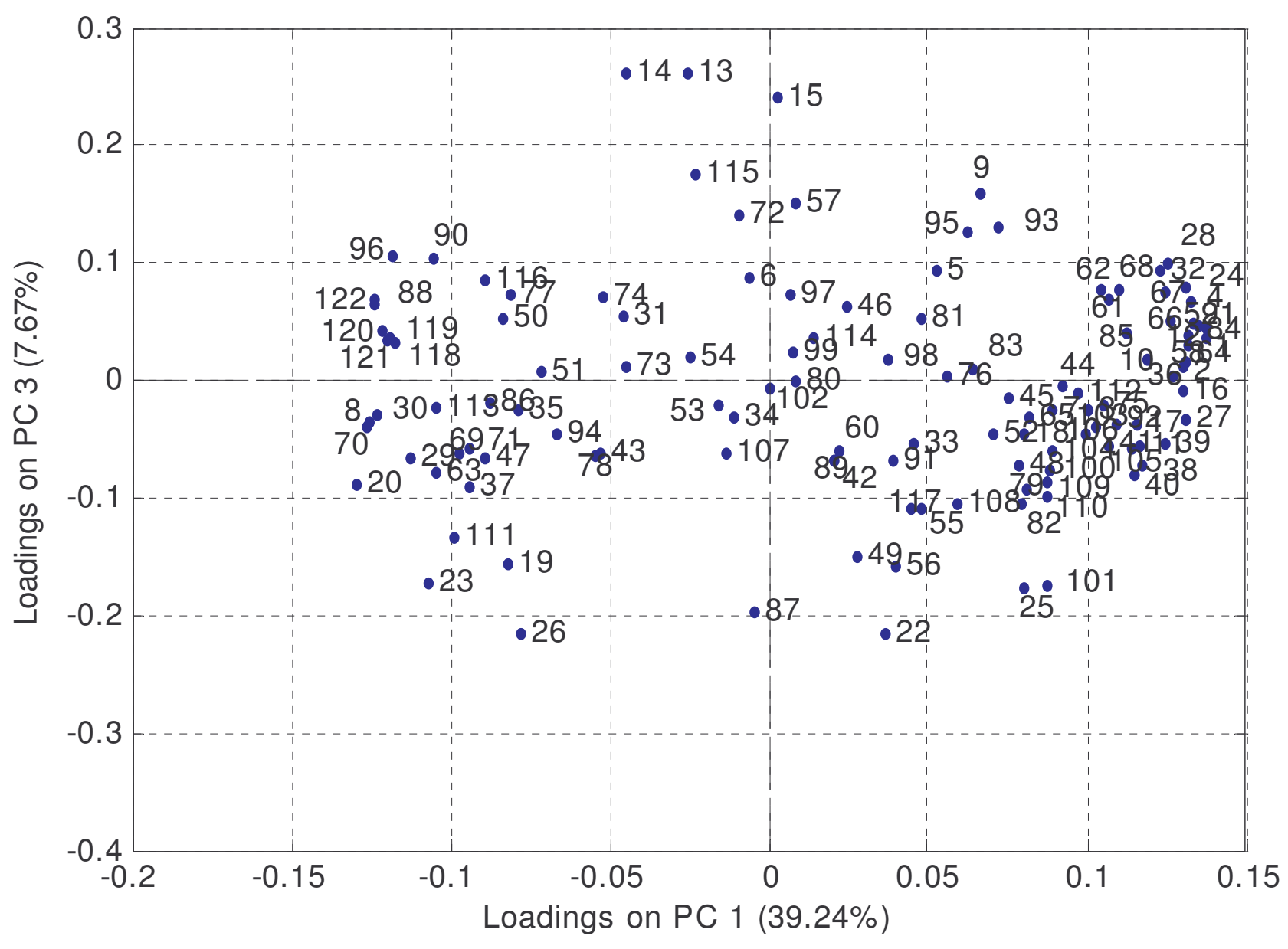


2D Loadings plot $p_{1}$ vs $p_{4}$

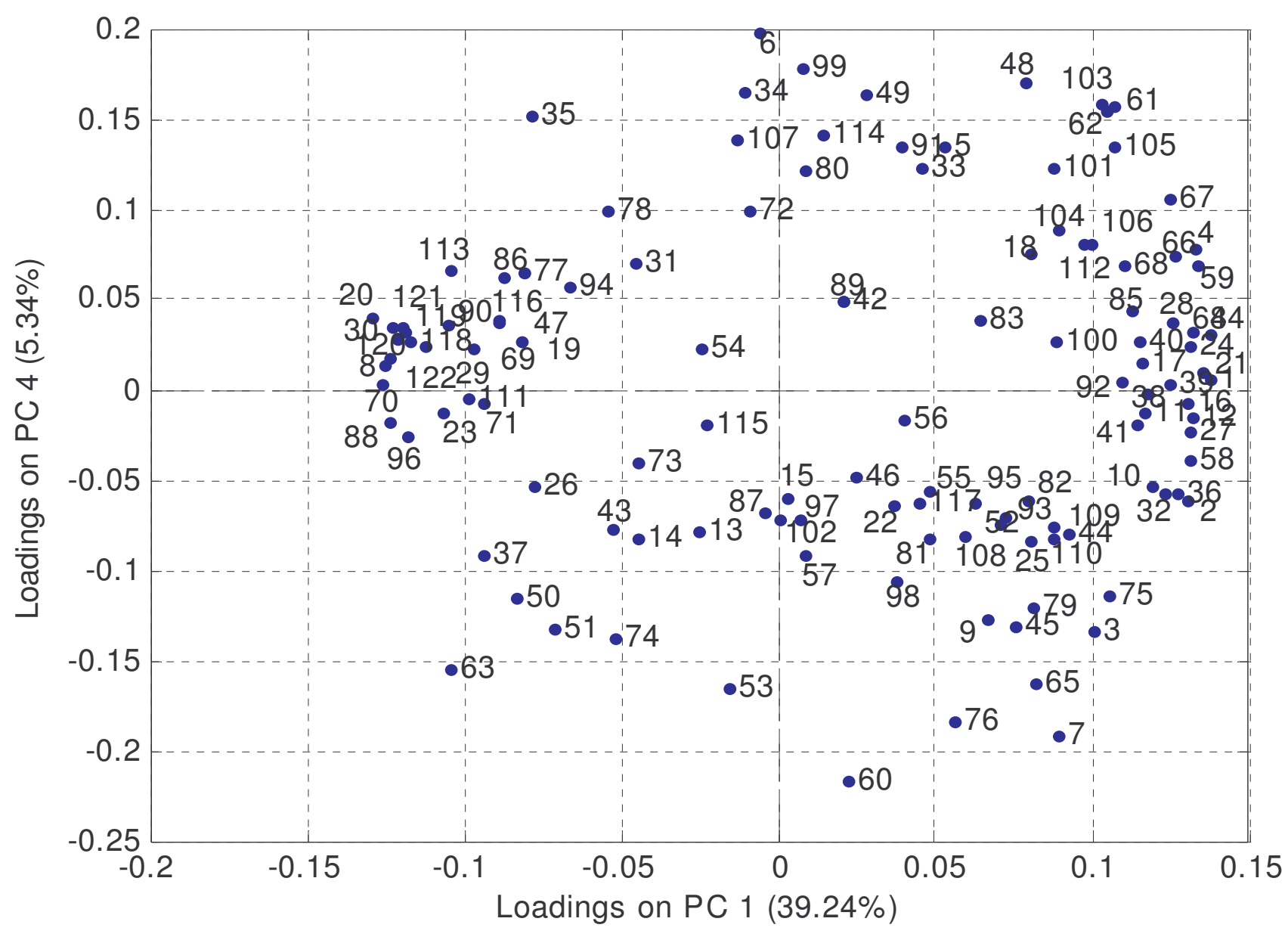


2D Loadings plot $p_{1}$ vs $p_{5}$

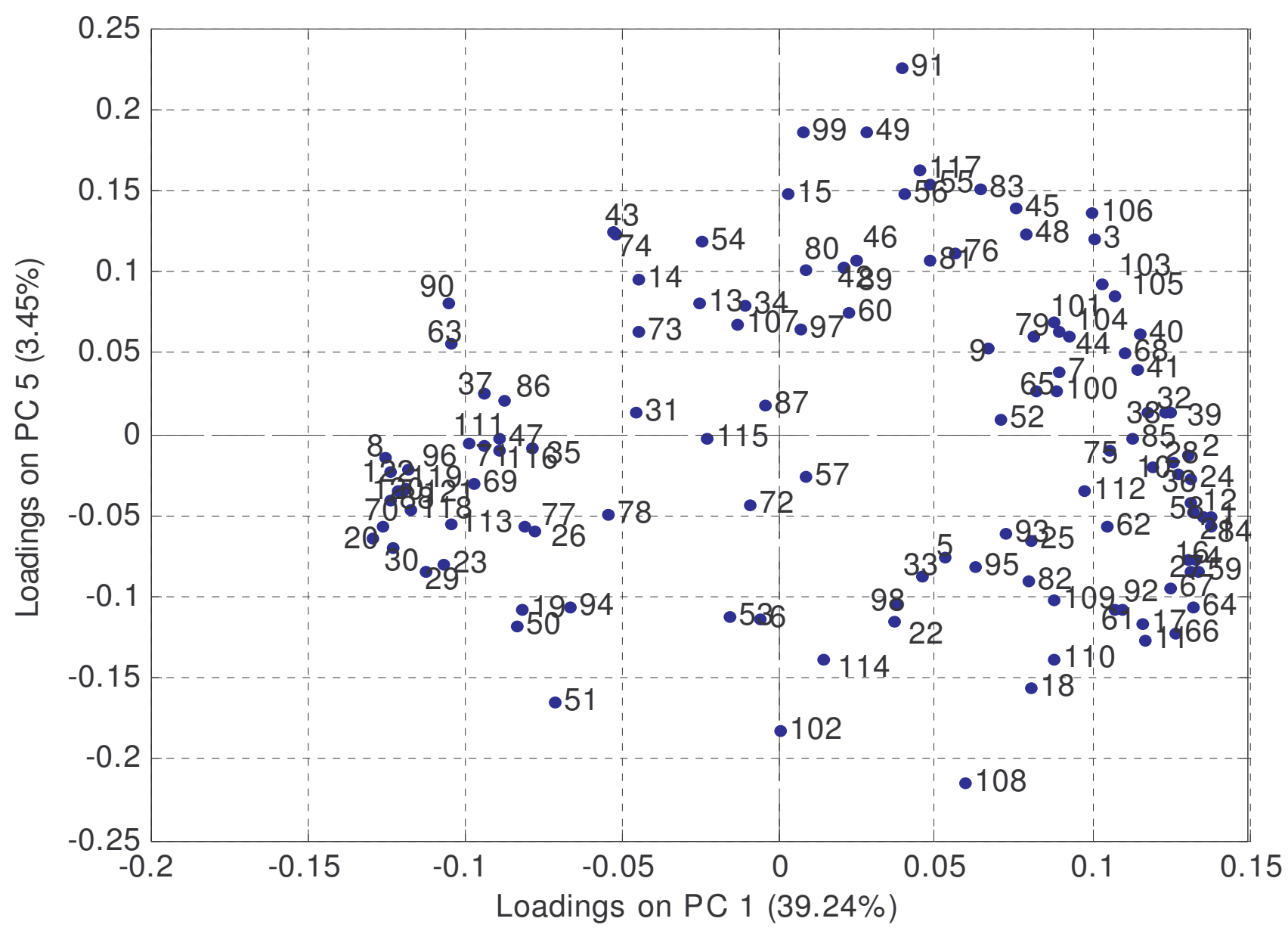


2D Loadings plot $p_{1}$ vs $p_{6}$

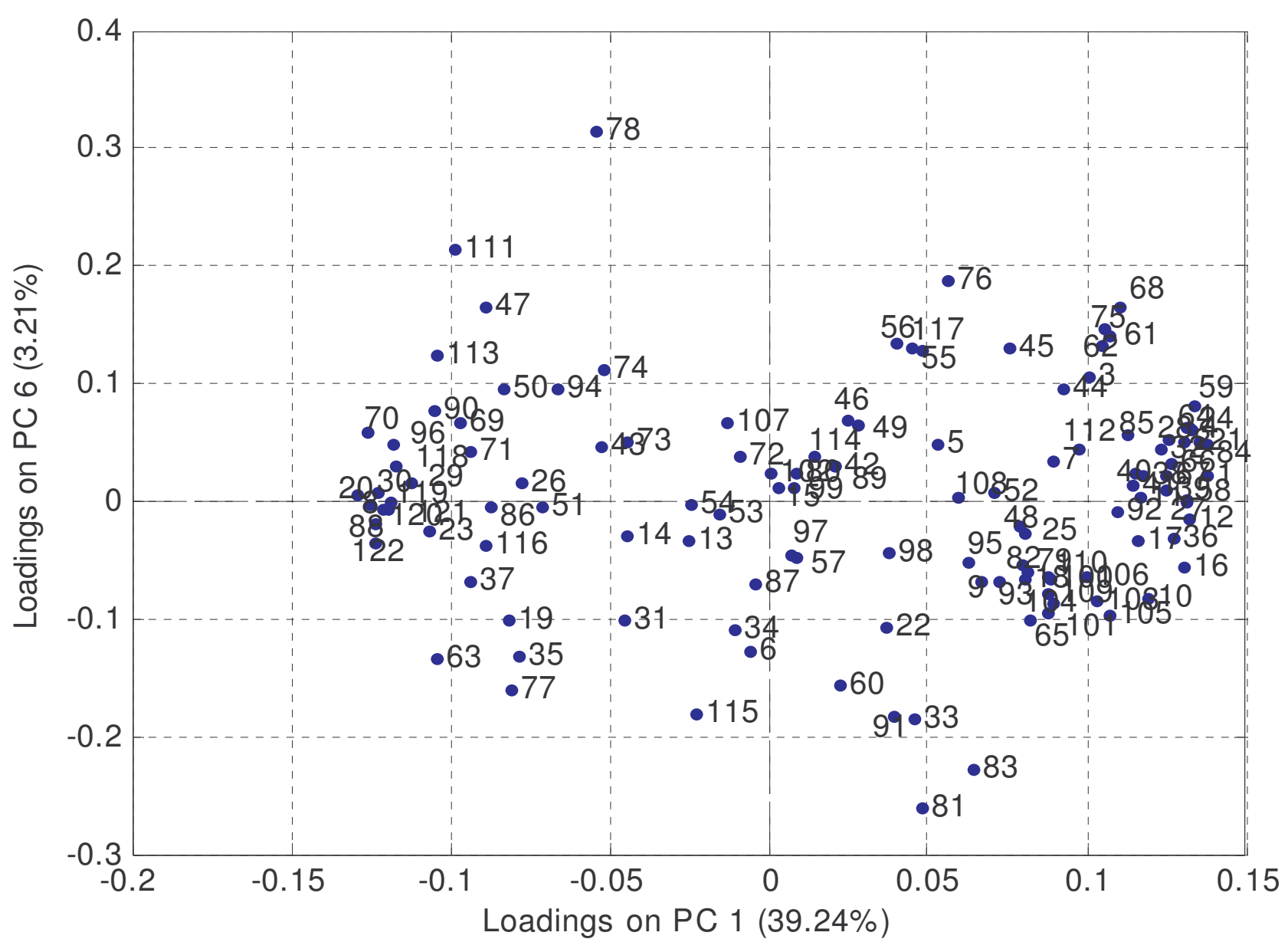


2D Loadings plot $p_{3}$ vs $p_{2}$

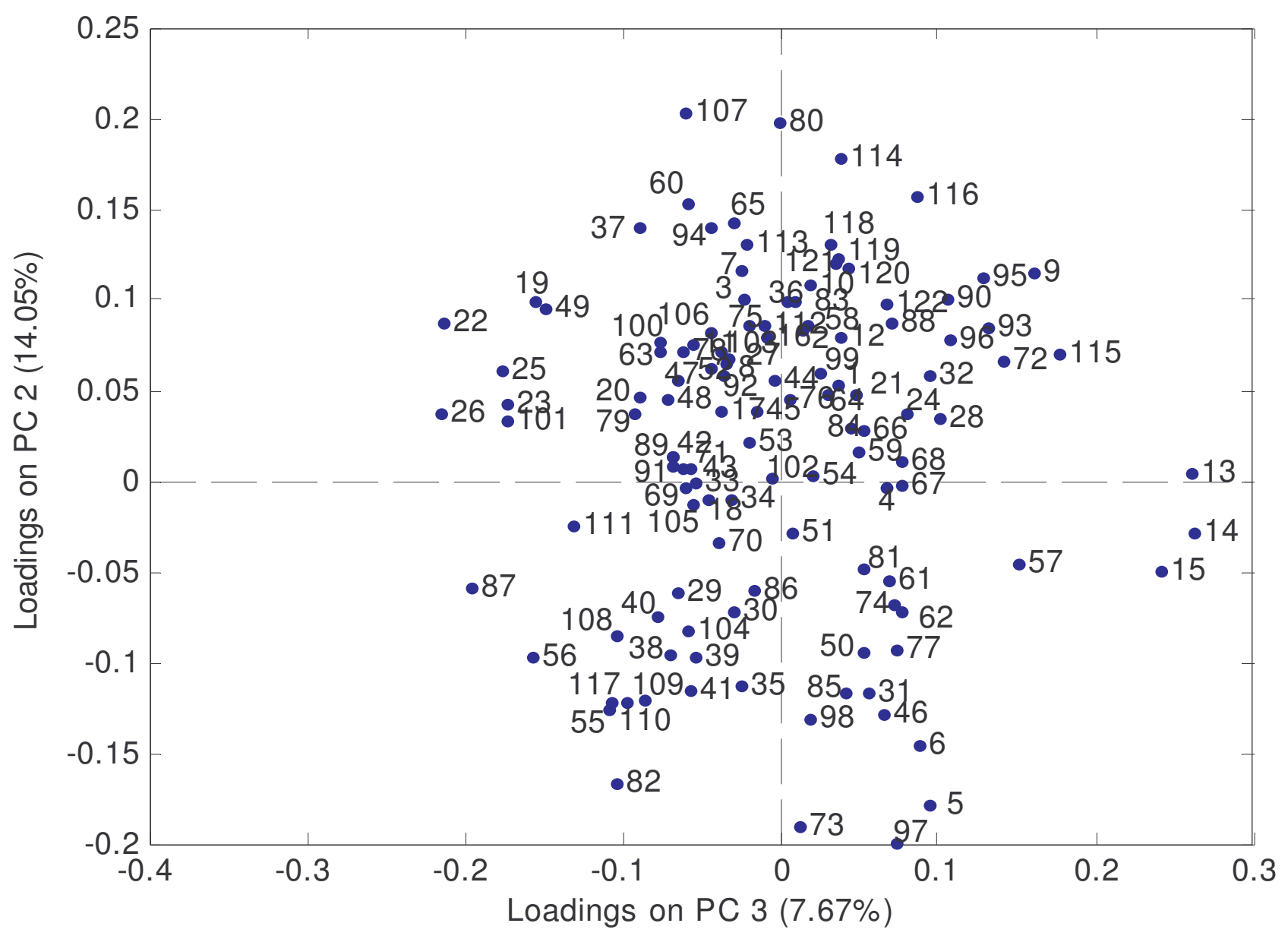


3D Loadings plot $p_{1}$ vs $p_{2}$ vs $p_{3}$

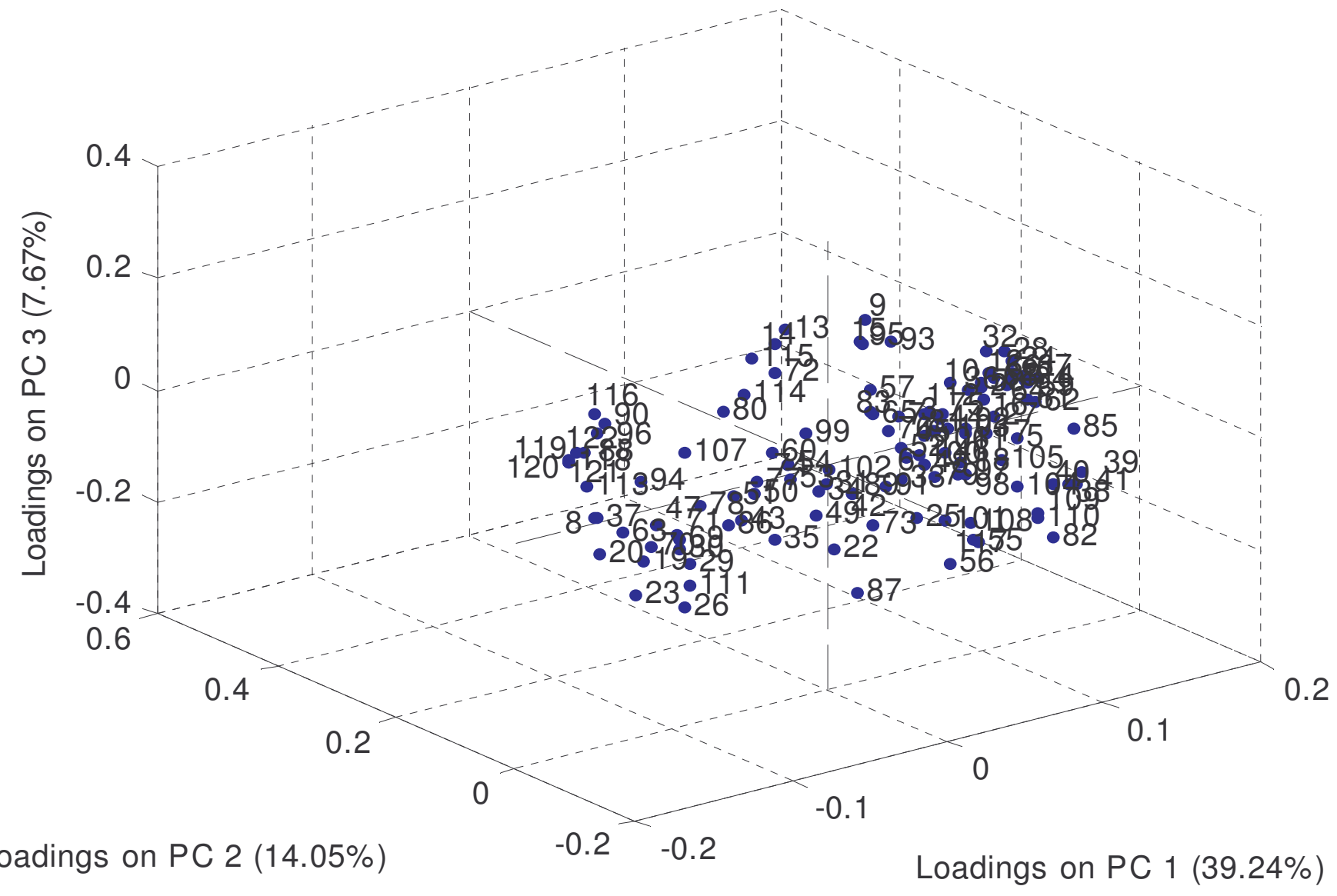


Table S6. Loading values achieved from the principal component analysis (PCA) of $\mathbf{X}_{77 \times 122}$.

\begin{tabular}{|c|c|c|c|c|c|c|c|c|c|c|}
\hline \multirow[b]{2}{*}{$\mathrm{md}^{\mathrm{a}}$} & \multicolumn{10}{|c|}{ Loadings from principal component analysis of the molecular descriptor matrix $\boldsymbol{X}_{77 \times 122}$} \\
\hline & $p_{1}$ & $\boldsymbol{p}_{2}$ & $p_{3}$ & $p_{4}$ & $p_{5}$ & $p_{6}$ & $p_{7}$ & $\boldsymbol{p}_{8}$ & $p_{9}$ & $\boldsymbol{p}_{10}$ \\
\hline 1 & 0.137 & 0.053 & 0.037 & 0.005 & -0.050 & 0.022 & -0.010 & 0.013 & 0.047 & 0.001 \\
\hline 2 & 0.130 & 0.084 & 0.013 & -0.061 & -0.012 & 0.051 & -0.026 & 0.033 & 0.063 & -0.024 \\
\hline 3 & 0.100 & 0.101 & -0.024 & -0.134 & 0.121 & 0.107 & -0.029 & -0.081 & 0.018 & -0.033 \\
\hline 4 & 0.132 & -0.004 & 0.068 & 0.078 & -0.077 & 0.061 & 0.006 & 0.027 & 0.024 & 0.005 \\
\hline 5 & 0.053 & -0.178 & 0.094 & 0.135 & -0.076 & 0.048 & 0.030 & 0.018 & 0.000 & -0.034 \\
\hline 6 & -0.006 & -0.146 & 0.088 & 0.198 & -0.113 & -0.128 & 0.010 & -0.041 & -0.034 & 0.047 \\
\hline 7 & 0.089 & 0.117 & -0.025 & -0.191 & 0.039 & 0.035 & -0.058 & 0.051 & 0.057 & -0.062 \\
\hline 8 & -0.125 & 0.065 & -0.034 & 0.014 & -0.014 & -0.001 & 0.001 & 0.130 & 0.012 & 0.073 \\
\hline 9 & 0.067 & 0.115 & 0.160 & -0.127 & 0.053 & -0.067 & -0.017 & -0.033 & 0.110 & 0.039 \\
\hline 10 & 0.119 & 0.109 & 0.018 & -0.053 & -0.020 & -0.082 & -0.030 & -0.013 & 0.086 & 0.026 \\
\hline 11 & 0.116 & 0.076 & -0.055 & -0.012 & -0.126 & 0.004 & -0.006 & 0.069 & 0.077 & 0.001 \\
\hline 12 & 0.132 & 0.079 & 0.039 & -0.015 & -0.048 & -0.014 & -0.011 & 0.011 & 0.046 & 0.033 \\
\hline 13 & -0.026 & 0.004 & 0.261 & -0.078 & 0.081 & -0.032 & 0.010 & 0.022 & 0.104 & 0.082 \\
\hline 14 & -0.045 & -0.029 & 0.261 & -0.083 & 0.096 & -0.028 & 0.073 & 0.044 & 0.045 & 0.060 \\
\hline 15 & 0.003 & -0.050 & 0.241 & -0.060 & 0.148 & 0.012 & 0.102 & -0.052 & -0.054 & 0.006 \\
\hline 16 & 0.130 & 0.080 & -0.008 & -0.008 & -0.077 & -0.055 & -0.017 & -0.035 & 0.060 & 0.032 \\
\hline 17 & 0.116 & 0.038 & -0.038 & 0.015 & -0.116 & -0.032 & -0.105 & -0.094 & 0.091 & 0.024 \\
\hline 18 & 0.080 & -0.010 & -0.046 & 0.076 & -0.156 & -0.066 & -0.124 & -0.141 & 0.098 & 0.061 \\
\hline 19 & -0.082 & 0.099 & -0.156 & 0.027 & -0.107 & -0.101 & 0.094 & -0.104 & -0.070 & -0.007 \\
\hline 20 & -0.129 & 0.047 & -0.089 & 0.040 & -0.063 & 0.006 & & & -0.016 & 0.006 \\
\hline 21 & 0.135 & 0.048 & 0.048 & 0.010 & -0.051 & 0.051 & -0.018 & & 0.061 & 0.002 \\
\hline 22 & 0.037 & 0.087 & -0.214 & -0.063 & -0.114 & -0.106 & & -0.034 & -0.138 & 0.004 \\
\hline 23 & -0.107 & 0.043 & -0.173 & -0.012 & -0.080 & -0.025 & & & -0.084 & 0.013 \\
\hline 24 & 0.131 & 0.038 & 0.081 & & -0.026 & 0.064 & -0. & & 0.084 & -0.001 \\
\hline 25 & 0.080 & 0.062 & -0.176 & -0.084 & -0.065 & -0.027 & & -0.028 & -0.162 & -0.019 \\
\hline 26 & -0.078 & 0.038 & -0.215 & -0. & -0.059 & 0.017 & 0. & 9 & -0.163 & -0.006 \\
\hline 27 & 0.131 & 0.068 & -0.033 & -0. & -0.084 & 0.001 & 0. & 5 & -0.013 & -0.002 \\
\hline 28 & 0.126 & 0.035 & 0.101 & 0.038 & -0.016 & 0.053 & -0 . & 0.026 & 0.109 & 0.006 \\
\hline 29 & -0.113 & -0.061 & -0.065 & 0.024 & -0.084 & 0.016 & & 74 & 0.124 & -0.039 \\
\hline 30 & -0.123 & -0.071 & -0.030 & 0.035 & -0.070 & 0.008 & -0.009 & 26 & 0.071 & -0.016 \\
\hline 31 & -0.046 & -0.116 & 0. & 0.070 & 0.014 & -0.100 & -0.002 & -0.132 & 0.030 & 0.154 \\
\hline 32 & 0.123 & 0.058 & 0.094 & -0.0 & 0.013 & 0.044 & -0.041 & 0.029 & 0.029 & -0.014 \\
\hline 33 & 0.046 & -0.001 & -0.053 & 0.123 & -0.087 & -0.184 & -0.030 & 0.185 & 0.190 & 0.122 \\
\hline 34 & -0.011 & -0.010 & -0.031 & 0.165 & 0.080 & -0.108 & -0.044 & -0.065 & -0.042 & -0.179 \\
\hline 35 & -0.079 & -0.113 & -0.025 & & -0.008 & -0.131 & -0.064 & 0.013 & 0.101 & 0.049 \\
\hline 36 & 0.127 & 0.099 & 0.004 & -0.058 & -0.024 & -0.031 & -0.022 & -0.010 & 0.071 & 0.003 \\
\hline 37 & -0.094 & 0.141 & -0.090 & -0.091 & 0.026 & -0.067 & -0.020 & 0.011 & 0.032 & 0.034 \\
\hline 38 & 0.117 & -0.096 & -0.071 & -0.002 & 0.014 & 0.023 & -0.004 & -0.013 & -0.071 & 0.045 \\
\hline 39 & 0.125 & -0.097 & -0.054 & 0.004 & 0.013 & 0.011 & 0.012 & 0.022 & -0.067 & 0.042 \\
\hline 40 & 0.115 & -0.074 & -0.079 & 0.028 & 0.062 & 0.024 & 0.186 & 0.015 & 0.066 & -0.030 \\
\hline 41 & 0.114 & -0.115 & -0.057 & -0.019 & 0.040 & 0.015 & 0.072 & 0.016 & -0.003 & -0.005 \\
\hline 42 & 0.020 & 0.014 & -0.068 & 0.050 & 0.103 & 0.031 & 0.361 & -0.007 & 0.250 & -0.133 \\
\hline 43 & -0.053 & 0.007 & -0.062 & -0.077 & 0.125 & 0.048 & -0.176 & -0.045 & -0.254 & -0.051 \\
\hline 44 & 0.092 & 0.056 & -0.004 & -0.079 & 0.061 & 0.095 & -0.057 & 0.061 & 0.068 & -0.011 \\
\hline 45 & 0.076 & 0.038 & -0.015 & -0.131 & 0.140 & 0.131 & -0.109 & -0.005 & 0.014 & -0.030 \\
\hline
\end{tabular}


Continuation of Table S6.

\begin{tabular}{|c|c|c|c|c|c|c|c|c|c|c|}
\hline \multirow[b]{2}{*}{$m d^{a}$} & \multicolumn{10}{|c|}{ Loadings from principal component analysis of the molecular descriptor matrix $\boldsymbol{X}_{77 \times 122}$} \\
\hline & $\begin{array}{c}\boldsymbol{p}_{1} \\
0.025\end{array}$ & $\begin{array}{c}\boldsymbol{p}_{2} \\
-0.129\end{array}$ & $\begin{array}{c}p_{3} \\
0.065\end{array}$ & $\begin{array}{c}\boldsymbol{p}_{4} \\
-0.048\end{array}$ & $\begin{array}{c}p_{5} \\
0.108\end{array}$ & $\begin{array}{c}p_{6} \\
0.069\end{array}$ & $\begin{array}{c}\boldsymbol{p}_{7} \\
-0.032\end{array}$ & $\begin{array}{c}p_{8} \\
0.035\end{array}$ & $\begin{array}{c}\boldsymbol{p}_{9} \\
-0.013\end{array}$ & $\begin{array}{c}\boldsymbol{p}_{10} \\
0.024\end{array}$ \\
\hline 47 & -0.089 & 0.056 & -0.065 & 0.038 & -0.003 & 0.165 & -0.039 & 0.099 & 0.114 & -0.019 \\
\hline 48 & 0.078 & 0.045 & -0.071 & 0.171 & & -0.022 & & 0.011 & 0.120 & 10 \\
\hline 49 & 0.028 & 0.096 & -0.149 & & & & & 0.012 & & -0.058 \\
\hline 50 & -0.083 & -0.094 & 0.053 & -0.115 & & & & & & 0.003 \\
\hline 51 & -0.071 & -0.028 & 0.008 & & & -0.005 & & -0.063 & -0.109 & 0.252 \\
\hline 52 & 0.071 & 0.063 & -0.044 & & & 0.007 & & -0.038 & 0.098 & 0.238 \\
\hline 53 & -0.016 & 0.022 & -0.020 & & & & & & 035 & 0.374 \\
\hline 54 & -0.025 & 0.003 & 0.021 & & & & & 0.071 & & -0.011 \\
\hline 55 & 48 & -0.126 & -0.109 & & & & & & & 0.162 \\
\hline 56 & & -0.097 & -0.157 & & & & & & & 0.271 \\
\hline 57 & & -0.045 & & & & & & & & -0.328 \\
\hline 58 & & 0.087 & 0.017 & & & & & & & \\
\hline 59 & & 0.017 & & & & & & & & \\
\hline 60 & & 0.153 & -0.059 & & & -0 . & & & & \\
\hline 61 & & -0.054 & & & & & & & & 0.000 \\
\hline 62 & & -0.072 & & & & & & & & 008 \\
\hline 63 & & 0.072 & -0.077 & & & -0. & & & & -0.008 \\
\hline 64 & & 0.048 & & & & & & & & 002 \\
\hline 65 & & 0.143 & -0.030 & & & -0 . & & & & -0.002 \\
\hline 66 & & 0.028 & & & & & & & & 037 \\
\hline 67 & & -0.002 & & & & & & & & 036 \\
\hline 68 & & 0.012 & & & & & & & & 0.069 \\
\hline 69 & 7 & -0.003 & -0.061 & & & & & & & 0.112 \\
\hline 7 & & -0.033 & -0.0 & & & & & & & -0.013 \\
\hline 71 & $-c$ & 0.007 & -0.057 & & & & & & & 038 \\
\hline 72 & & & & & & & & & & 0.190 \\
\hline 73 & & -0.190 & & & & & & & & 0.018 \\
\hline 74 & -0 & -0.068 & & & & & & & & 0.006 \\
\hline 75 & & 0.086 & -0.021 & & & & & & & -0.015 \\
\hline 76 & & & & & & & & & & -0.078 \\
\hline 77 & & -0.093 & & & & -0 . & & & & 0.000 \\
\hline 78 & & 0.071 & -0.063 & & & & & & 40 & -0.003 \\
\hline 79 & & 0.037 & -0.093 & & & -0.060 & & & 0.007 & 0.040 \\
\hline 80 & & 0.198 & & & & & & & & 0.052 \\
\hline 81 & & -0.047 & & & & -0.2 & & 0.209 & -0.114 & 0.117 \\
\hline 82 & & -0.166 & -0.104 & -0 & & -0. & & 0.032 & 0.030 & -0.049 \\
\hline 83 & & 0.099 & & & & -0.228 & & 0.168 & -0.138 & 0.128 \\
\hline 84 & & 0.030 & & & & & & 0.023 & 0.038 & -0.008 \\
\hline 85 & & -0.116 & & & & & & -0.089 & -0 . & -0.091 \\
\hline 86 & & -0.060 & -0. & & & -0. & & 0.248 & & 0.011 \\
\hline 87 & -0.004 & -0.058 & -0. & -0. & & -0. & & & -0.147 & -0.118 \\
\hline 88 & -0.12 & 0.087 & & -0. & & -0. & & & -0.005 & -0.003 \\
\hline 89 & & 0.014 & -0.068 & & & & & & 0.250 & -0.133 \\
\hline 90 & -0.106 & 0.100 & 0.105 & 0.036 & 0.081 & 0.078 & 0.097 & -0.087 & -0.011 & 0.007 \\
\hline
\end{tabular}


Continuation of Table S6.

\begin{tabular}{|c|c|c|c|c|c|c|c|c|c|c|}
\hline \multirow[b]{2}{*}{$\mathrm{md}^{\mathrm{a}}$} & \multicolumn{10}{|c|}{ Loadings from principal component analysis of the molecular descriptor matrix $\boldsymbol{X}_{77 \times 122}$} \\
\hline & $p_{1}$ & $p_{2}$ & $p_{3}$ & $p_{4}$ & $p_{5}$ & $p_{6}$ & $p_{7}$ & $p_{8}$ & $p_{9}$ & $\boldsymbol{p}_{10}$ \\
\hline 91 & 0.039 & 0.008 & -0.068 & 0.134 & 0.226 & -0.183 & -0.105 & -0.113 & 0.001 & 0.089 \\
\hline 92 & 0.109 & 0.059 & -0.037 & 0.005 & -0.108 & -0.009 & -0.018 & 0.127 & -0.049 & -0.011 \\
\hline 93 & 0.072 & 0.085 & 0.131 & -0.070 & -0.060 & -0.067 & 0.215 & 0.187 & -0.027 & -0.036 \\
\hline 94 & -0.067 & 0.140 & -0.045 & 0.057 & -0.106 & 0.097 & -0.028 & -0.120 & -0.090 & -0.053 \\
\hline 95 & 0.063 & 0.113 & 0.128 & -0.062 & -0.081 & -0.052 & 0.217 & 0.172 & -0.044 & -0.047 \\
\hline 96 & -0.118 & 0.078 & 0.107 & -0.026 & -0.021 & 0.049 & 0.010 & -0.043 & 0.035 & 0.046 \\
\hline 97 & 0.007 & -0.199 & 0.074 & -0.072 & 0.064 & -0.044 & 0.082 & -0.003 & 0.066 & 0.083 \\
\hline 98 & 0.037 & -0.131 & 0.019 & -0.106 & -0.105 & -0.042 & 0.167 & 0.167 & -0.033 & -0.045 \\
\hline 99 & 0.007 & 0.060 & 0.025 & 0.179 & 0.187 & 0.012 & 0.268 & 0.109 & -0.039 & 0.073 \\
\hline 100 & 0.088 & 0.077 & -0.076 & 0.027 & 0.027 & -0.065 & -0.097 & 0.016 & -0.008 & -0.072 \\
\hline 101 & 0.088 & 0.034 & -0.173 & 0.123 & 0.070 & -0.094 & 0.122 & -0.040 & 0.034 & -0.034 \\
\hline 102 & 0.000 & 0.002 & -0.005 & -0.072 & -0.182 & 0.024 & 0.132 & -0.040 & -0.140 & 0.315 \\
\hline 103 & 0.103 & 0.072 & -0.038 & 8 & 0.093 & -0.084 & -0.074 & 0.0 & -0.009 & 0.095 \\
\hline 104 & 0.089 & -0.082 & -0.060 & 0. & 0.063 & -0.087 & -0.110 & -0.016 & 0.056 & 0.076 \\
\hline 105 & 0.107 & -0.012 & -0.056 & 0.1 & 0.086 & -0.095 & -0.105 & 0.019 & 0.029 & 0.095 \\
\hline 106 & 0.100 & 0.082 & -0.044 & 0.081 & 0.137 & -0.063 & 0.127 & 0.089 & -0.151 & 0.018 \\
\hline 107 & -0.014 & 0.204 & -0.061 & 0.139 & 0.069 & 0.066 & 0.074 & 0.013 & -0.076 & 0.054 \\
\hline 108 & 0.059 & -0.085 & -0.104 & -0.080 & -0.214 & 0.004 & 0.040 & 0.039 & 0.049 & -0.071 \\
\hline 109 & 0.087 & -0.121 & -0.087 & -0.076 & -0.102 & -0.077 & -0.029 & 0.068 & 0.048 & -0.099 \\
\hline 110 & 0.087 & -0.122 & -0.098 & -0.083 & -0.137 & -0.063 & -0.015 & 0.066 & 0.052 & -0.099 \\
\hline 111 & -0.099 & -0.024 & -0.132 & -0.004 & -0.005 & 0.214 & -0.026 & -0.070 & 0.108 & 0.034 \\
\hline 112 & 0.097 & 0.087 & -0.010 & 31 & -0.034 & 0.046 & -0.133 & 0.065 & -0.099 & 0.113 \\
\hline 113 & -0.104 & 0.132 & -0.021 & 0.067 & -0.055 & 0.124 & -0.040 & -0.068 & 0.060 & 0.038 \\
\hline 114 & 0.014 & 0.178 & 0.037 & 0.141 & -0.138 & 0.039 & -0.084 & 0.027 & -0.057 & 0.028 \\
\hline 115 & -0.023 & 0.070 & 0.176 & -0.020 & -0.003 & -0.180 & 0.110 & -0.122 & -0.113 & -0.077 \\
\hline 116 & -0.090 & 0.158 & 0.086 & 0.039 & -0.009 & -0.037 & 0.007 & -0.064 & 0.024 & 0.015 \\
\hline 117 & 0.045 & -0.122 & -0.108 & -0.062 & 0.163 & 0.131 & 0.032 & -0.115 & 0.023 & 0.168 \\
\hline 118 & -0.117 & 0.131 & 0.032 & 0.027 & -0.047 & 0.030 & -0.027 & -0.029 & 0.035 & -0.011 \\
\hline 119 & -0.119 & 0.123 & 0.036 & 0.032 & -0.033 & 0.000 & -0.022 & -0.016 & 0.045 & -0.014 \\
\hline 120 & -0.121 & 0.117 & 0.043 & 0.028 & -0.034 & -0.006 & -0.017 & -0.016 & 0.046 & -0.010 \\
\hline 121 & 0.039 & 0.008 & -0.068 & 0.134 & 0.226 & -0.183 & -0.105 & -0.113 & 0.001 & 0.089 \\
\hline 122 & 0.109 & 0.059 & -0.037 & 0.005 & -0.108 & -0.009 & -0.018 & 0.127 & -0.049 & -0.011 \\
\hline
\end{tabular}

